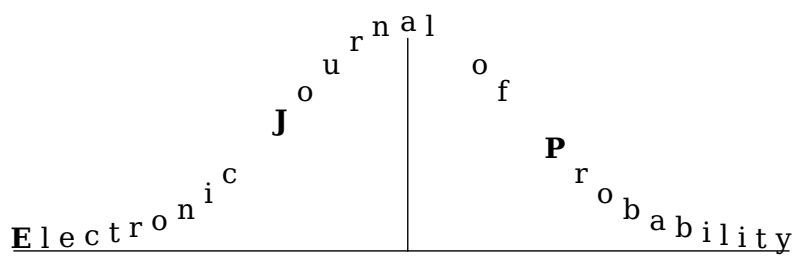

Electron. J. Probab. 26 (2021), article no. 160, 1-22.

ISSN: 1083-6489 https://doi.org/10.1214/21-EJP727

\title{
Gaussian fluctuations and a law of the iterated logarithm for Nerman's martingale in the supercritical general branching process*
}

\author{
Alexander Iksanov ${ }^{\dagger} \quad$ Konrad Kolesko $^{\ddagger \S} \quad$ Matthias Meiners $^{\ddagger}$
}

\begin{abstract}
In his, by now, classical work from 1981, Nerman made extensive use of a crucial martingale $\left(W_{t}\right)_{t \geq 0}$ to prove convergence in probability, in mean and almost surely, of supercritical general branching processes (also known as Crump-Mode-Jagers branching processes) counted with a general characteristic. The martingale terminal value $W$ figures in the limits of his results.

We investigate the rate at which the martingale, now called Nerman's martingale, converges to its limit $W$. More precisely, assuming the existence of a Malthusian parameter $\alpha>0$ and $W_{0} \in L^{2}$, we prove a functional central limit theorem for $\left(W-W_{t+s}\right)_{s \in \mathbb{R}}$, properly normalized, as $t \rightarrow \infty$. The weak limit is a randomly scaled time-changed Brownian motion. Under an additional technical assumption, we prove a law of the iterated logarithm for $W-W_{t}$.
\end{abstract}

Keywords: asymptotic fluctuations; functional central limit theorem; law of the iterated logarithm; Nerman's martingale; supercritical general branching process.

MSC2020 subject classifications: 60J80; 60F05; 60F17.

Submitted to EJP on July 1, 2021, final version accepted on November 26, 2021.

\section{Introduction}

The general (Crump-Mode-Jagers) branching process is a classical model for an evolving population. The process starts with one initial ancestor at time 0 which produces

\footnotetext{
*A. Iksanov was partially supported by the grant ME3625/3-1 and supported by Ulam programme funded by the Polish national agency for academic exchange (NAWA), project no. PPN/ULM/2019/1/00010/DEC/1. K. Kolesko was partially supported by the National Science Center, Poland (Sonata Bis, grant number DEC2014/14/E/ST1/00588).

${ }^{\dagger}$ Faculty of Computer Science and Cybernetics, Taras Shevchenko National University of Kyiv, Ukraine.

E-mail: iksan@univ.kiev.ua

${ }^{\ddagger}$ Mathematisches Institut, University of Gießen, Germany.

E-mail: konrad.kolesko@math.uni-giessen.de

E-mail: matthias.meiners@math .uni-giessen.de

${ }_{\S}^{\S}$ Mathematical Institute, University of Wrocław, Poland.
} 
offspring at the times of a point process $\xi$ on $(0, \infty)$. Every other individual in the process reproduces according to an independent copy of $\xi$ shifted by the individual's time of birth. The model contains a variety of other models such as Galton-Watson processes, age-dependent branching processes, Bellman-Harris processes and Sevast'yanov processes. Counted with a random characteristic the general branching process offers a lot of flexibility in modelling and allows considerations of, for instance, the number of individuals in the population in some random phase of life or having some random age-dependent property. We refer to [17] for a textbook introduction.

The weak and strong laws of large numbers for supercritical general branching processes counted with a random characteristic were given by Nerman [22]. Assuming the existence of some $\alpha>0$ such that $m(\alpha)=1$ where $m$ is the Laplace transform of the intensity measure of the reproduction point process $\xi$, these laws exhibit exponential growth of the order $e^{\alpha t}$ of the process, i.e., $\alpha$ is a Malthusian exponent. Key to the proof of these results is a crucial martingale $\left(W_{t}\right)_{t>0}$, nowadays called Nerman's martingale. The martingale limit $W$ appears in the aforementioned weak and strong laws. Further, the rate of convergence of the martingale to its limit is relevant for the rate of convergence in the weak law of large numbers for the general branching process [16].

Recently, Janson [19] studied the fluctuations of supercritical general branching processes in the case where $\xi$ is concentrated on a lattice. A natural first step towards extending Janson's results to the non-lattice case is to investigate the asymptotic fluctuations of Nerman's martingale around its limit. In the paper at hand, we address this problem by proving a functional central limit theorem with a deterministic scaling for $W-W_{t}$. This functional limit theorem is complemented by a law of the iterated logarithm.

The results of the present paper are analogous to those for Biggins' martingale in the branching random walk [13]. Fluctuations of the latter martingale, also at complex parameters, have received a lot of attention lately [14, 15, 24]. The endmost paper [24] is in the more general context of weighted branching processes. Predecessors of these results are central limit theorems for the classical martingale in the Galton-Watson process $[10,11,12]$. There are further rate-of-convergence results for multitype GaltonWatson processes, we refrain from providing references here and refer to the discussion in [21] instead.

Closely related to Biggins' martingale in the branching random walk is the derivative martingale, the fluctuations of which have been addressed in [6]. The counterpart for the derivative martingale in branching Brownian motion is contained in [21]. Rate-ofconvergence results for more complicated branching processes, including branching diffusions and superprocesses, can be found in [23] and the references therein.

\section{Model and assumptions}

We begin by introducing the standard Ulam-Harris notation in the context of the general branching process. We mainly follow [18]. Let $\mathcal{I}:=\bigcup_{n \in \mathbb{N}_{0}} \mathbb{N}^{n}$ be the infinite Ulam-Harris tree where $\mathbb{N}=\{1,2, \ldots\}, \mathbb{N}_{0}=\mathbb{N} \cup\{0\}$, and $\mathbb{N}^{0}=\{\varnothing\}$ contains only the empty tuple, which we denote by $\varnothing$. We identify individuals in a population with their descent, which is encoded by elements of $\mathcal{I}$. For instance, $\varnothing$ is the label of the ancestor, and if $u=\left(u_{1}, \ldots, u_{m}\right) \in \mathcal{I}$, then $u$ is the $u_{m}$ th child of the $u_{m-1}$ th child of $\ldots$ of the $u_{1}$ th child of the ancestor $\varnothing$. We abbreviate $u=\left(u_{1}, \ldots, u_{m}\right)$ by $u_{1} \ldots u_{m}$ and set $|u|$ for the generation of $u$. Here, $|u|=m$. Similarly, if $v=\left(v_{1}, \ldots, v_{n}\right)$, we write $u v$ for $\left(u_{1}, \ldots, u_{m}, v_{1}, \ldots, v_{n}\right)$. Further, if $k \leq m$, we write $\left.u\right|_{k}$ for $u_{1} \ldots u_{k}$, the ancestor of $u$ in the $k$ th generation. If $u=\left.v\right|_{m}$ for some $0 \leq m \leq|v|$, i.e., when $u$ is an ancestor of $v$, then we write $u \preceq v$, and say that $v$ stems from $u$. For a subset $F$ of $\mathcal{I}$, we say that $v$ stems 
from $F$, and in this case write $F \preceq v$, if $u \preceq v$ for some $u \in F$. For $F, L \subseteq \mathcal{I}$, we write $F \preceq L$ if every $v \in L$ stems from some $u \in F$.

\subsection{The model}

Let $(\Omega, \mathcal{F}, \mathbb{P})$ be a probability space on which a family $\left(\xi_{u}\right)_{u \in \mathcal{I}}$ of independent, identically distributed (i.i.d.) point processes on $(0, \infty)$ is defined. Formally, each $\xi_{u}$ is an integer-valued mapping $\xi_{u}: \Omega \times \mathcal{B}((0, \infty)) \rightarrow[0, \infty]$ such that

- for fixed $\omega \in \Omega, \xi_{u}(\omega, \cdot): \mathcal{B}((0, \infty)) \rightarrow[0, \infty]$ is a measure,

- whereas, for each Borel set $B \in \mathcal{B}((0, \infty))$, the map $\xi_{u}(\cdot, B): \Omega \rightarrow[0, \infty]$ is a random variable.

Here, $\mathcal{B}((0, \infty))$ is the Borel $\sigma$-algebra of $(0, \infty)$. We write $\xi_{u}=\sum_{k=1}^{N(u)} \delta_{X_{k}(u)}$ for $u \in \mathcal{I}$ where $N(u):=\xi_{u}((0, \infty))$ is a random variable taking values in $\mathbb{N}_{0} \cup\{\infty\}$. For convenience, we abbreviate $\xi_{\varnothing}$ to $\xi, N(\varnothing)$ to $N, X_{k}(\varnothing)$ to $X_{k}$ etc. Further, we define $S(u)$, the time of birth of individual $u$, recursively via

$$
S(\varnothing):=0 \quad \text { and } \quad S(u k):=S(u)+X_{k}(u) \quad \text { for } u \in \mathcal{I} \text { and } k \in \mathbb{N}
$$

with the convention that $X_{k}(u):=\infty$ if $k>N(u)$. If $u \in \mathcal{I}$ with $S(u)=\infty$, then individual $u$ is considered never born. We define $\mathcal{G}_{n}:=\left\{u \in \mathbb{N}^{n}: S(u)<\infty\right\}$ to be the $n$th generation individuals, $n \in \mathbb{N}_{0}$. Further, $\mathcal{G}:=\bigcup_{n \in \mathbb{N}_{0}} \mathcal{G}_{n}$ denotes the set of all individuals that are ever born. We set

$$
\mathcal{I}_{t}:=\left\{u \in \mathcal{I}: S\left(\left.u\right|_{|u|-1}\right) \leq t<S(u)<\infty\right\}
$$

to be the coming generation at time $t$, that is, $\mathcal{I}_{t}$ is the set of particles born after $t$ whose parents were born at or before time $t$.

Later on, different filtrations will be important. We call a subset $L \subseteq \mathcal{I}$ a line if $u \npreceq v$ for all $u, v \in L$ with $u \neq v$. For any line $L$, we define $\mathcal{F}_{L}:=\sigma\left(\xi_{u}: L \npreceq u\right)$. The $\sigma$-algebra $\mathcal{F}_{L}$ contains all information about the individuals up to and including the line $L$ in the genealogical tree, but is independent of all information that comes after that, i.e., after crossing the line $L$, for instance, the relative birth times of individuals descended from an element of $L$. Of particular importance are the $\sigma$-algebras with $L=\mathbb{N}^{n}$, the $n$th generation,

$$
\mathcal{F}_{n}:=\mathcal{F}_{\mathbb{N}^{n}}=\sigma\left(\xi_{u}:|u|<n\right), \quad n \in \mathbb{N}_{0} .
$$

The family $\left(\mathcal{F}_{n}\right)_{n \in \mathbb{N}_{0}}$ forms a filtration of $(\Omega, \mathcal{F})$. We set $\mathcal{F}_{\infty}:=\sigma\left(\mathcal{F}_{n}: n \in \mathbb{N}\right)$. The second filtration is the counterpart of the first when the $n$th generation is replaced by the coming generation at time $t$. To formally introduce it, we first recall the notion of an optional line. An optional line $\mathcal{J} \subseteq \mathcal{I}$ is a random line with the property that, for every deterministic line $L \subseteq \mathcal{I}$, it holds that $\{\mathcal{J} \preceq L\} \in \mathcal{F}_{L}$. For instance, for every $t \geq 0$, the coming generation at time $t, \mathcal{I}_{t}$, is an optional line. Indeed, for every line $L \subseteq \mathcal{I}$, we have

$$
\left\{\mathcal{I}_{t} \preceq L\right\}=\bigcap_{v \in L}\left\{\mathcal{I}_{t} \preceq v\right\}=\bigcap_{v \in L}\{S(v)>t\} \in \mathcal{F}_{L} .
$$

We follow Jagers on p. 190 of [18] and define, for an optional line $\mathcal{J}$,

$$
\mathcal{F}_{\mathcal{J}}:=\left\{A \in \mathcal{F}_{\infty}: A \cap\{\mathcal{J} \preceq L\} \in \mathcal{F}_{L} \text { for all lines } L \subseteq \mathcal{I}\right\} .
$$

A key result for us is the strong Markov branching property at optional lines, Theorem 4.14 in [18]. Finally, for $t \geq 0$, we set $\mathcal{H}_{t}:=\mathcal{F}_{\mathcal{I}_{t}}$. 


\section{Gaussian fluctuations of Nerman's martingale}

\subsection{Basic assumptions}

Let $\mu(\cdot):=\mathbb{E}[\xi(\cdot)]$ be the intensity measure of the reproduction point processes. It is a measure on $\mathcal{B}((0, \infty))$. Throughout the paper, we assume that $\mu$ is not concentrated on any lattice $h \mathbb{N}_{0}, h>0$. This assumption is for convenience only, all results have lattice counterparts. We define

$$
m(\theta):=\int_{(0, \infty)} e^{-\theta t} \mu(\mathrm{d} t), \quad \theta \geq 0 .
$$

The function $m$ is the Laplace transform of the intensity measure $\mu$.

Consider the following assumptions:

(A1) The process is supercritical, i.e., $\mathbb{E}[N]=\mu((0, \infty))>1$.

(A2) There exists a Malthusian parameter $\alpha>0$, i.e., an $\alpha>0$ satisfying

$$
m(\alpha)=\int_{(0, \infty)} e^{-\alpha t} \mu(\mathrm{d} t)=1
$$

(A3) The (right) derivative at $\theta=\alpha$ of the Laplace transform $m$ is finite, i.e.,

$$
m^{\prime}(\alpha):=-\int_{(0, \infty)} t e^{-\alpha t} \mu(\mathrm{d} t) \in(-\infty, 0) .
$$

(A4) The (random) Laplace transform of $\xi$ at $\theta=\alpha$ has positive and finite variance, i.e.,

$$
0<\sigma^{2}:=\mathbb{E}\left[\left(\sum_{k=1}^{N} e^{-\alpha X_{k}}-1\right)^{2}\right]=\mathbb{E}\left[\left(\int_{(0, \infty)} e^{-\alpha t} \xi(\mathrm{d} t)-1\right)^{2}\right]<\infty
$$

(A5) There exists a nonincreasing Lebesgue integrable function $g:[0, \infty) \rightarrow(0, \infty)$ such that

$$
\mathbb{E}\left[\sup _{t \geq 0} \frac{\sum_{k=1}^{N} e^{-\alpha X_{k}} \mathbb{1}_{\left[0, X_{k}\right)}(t)}{g(t)}\right]<\infty .
$$

Throughout the paper, we shall assume that (A1) through (A3) hold. Assumption (A1) guarantees that the survival set $\mathcal{S}$ defined by

$$
\mathcal{S}=\left\{\mathcal{G}_{n} \neq \varnothing \text { for all } n \in \mathbb{N}_{0}\right\}
$$

satisfies $\mathrm{P}(\mathcal{S})>0$. While assumption (A4) is required for the central limit theorem for Nerman's martingale, (A5) is additionally used in the proof of the law of the iterated logarithm.

\subsection{Nerman's martingale}

Recall that $\mathcal{I}_{t}$ is the coming generation at time $t$, i.e., the collection of labels of individuals born after time $t$ whose parents were born up to (and including) time $t$. Put

$$
W_{t}:=\sum_{u \in \mathcal{I}_{t}} e^{-\alpha S(u)}, \quad t \geq 0 .
$$

The family $\left(W_{t}, \mathcal{H}_{t}\right)_{t \geq 0}$ is a nonnegative martingale (Proposition 2.4 in [22]), called Nerman's martingale. It converges almost surely (a.s.) as $t \rightarrow \infty$ to a finite limit $W \geq 0$ (Corollary 2.5 in [22]). For later use, we stipulate $W_{t}:=1$ for $t<0$. The martingale 


\section{Gaussian fluctuations of Nerman's martingale}

is a pure jump process almost surely taking values in the Skorokhod space $D(\mathbb{R})$ of right-continuous real-valued functions with left limits at every point (càdlàg functions). With probability one, there are only finitely many jumps on every given compact set. Indeed, the martingale jumps at $t \geq 0$ only if, for some $n \in \mathbb{N}$, there are $u_{1}, \ldots, u_{n} \in \mathcal{G}$ and $S\left(u_{j}\right)=t$ for $j=1, \ldots, n$. In this case

$$
\Delta W_{t}:=W_{t}-W_{t-}=\sum_{j=1}^{n} e^{-\alpha S\left(u_{j}\right)}\left(\sum_{k \geq 1} e^{-\alpha X_{k}\left(u_{j}\right)}-1\right) .
$$

Almost surely, there are only finitely many $u$ with $S(u) \leq t$ for any $t \geq 0$. Indeed, by the many-to-one lemma [25, Section 1.3], there is a zero-delayed random walk $\left(\mathrm{S}_{n}\right)_{n \in \mathbb{N}_{0}}$ on $\mathbb{R}$ with increment law $\mathbb{P}\left(\mathrm{S}_{n}-\mathrm{S}_{n-1} \in \mathrm{d} x\right)=e^{-\alpha x} \mu(\mathrm{d} x), \mathbb{E}\left[\mathrm{S}_{1}\right]=-m^{\prime}(\alpha)>0$ and

$$
\begin{aligned}
\mathbb{E}\left[N_{t}\right] & =\sum_{n \geq 0} \mathbb{E}\left[\sum_{|u|=n} \mathbb{1}_{\{S(u) \leq t\}}\right] \leq e^{\alpha t} \sum_{n \geq 0} \mathbb{E}\left[\sum_{|u|=n} e^{-\alpha S(u)} \mathbb{1}_{\{S(u) \leq t\}}\right] \\
& =e^{\alpha t} \sum_{n \geq 0} \mathbb{P}\left(\mathrm{S}_{n} \in(0, t]\right)<\infty
\end{aligned}
$$

The process $\left(N_{t}\right)_{t \geq 0}$ is also a natural example of a process the limit of which is given by a constant multiple of Nerman's martingale. Indeed, if (A1) through (A5) hold ((A4) is not needed), Theorem 5.4 of [22] implies that $e^{-\alpha t} N_{t} \rightarrow \frac{1}{-\alpha m^{\prime}(\alpha)} W$ a. s. as $t \rightarrow \infty$.

\subsection{The connection with Biggins' martingale}

Nerman's martingale is related to the corresponding Biggins martingale $\left(Z_{n}, \mathcal{F}_{n}\right)_{n \in \mathbb{N}_{0}}$, where

$$
Z_{n}=\sum_{|u|=n} e^{-\alpha S(u)}, \quad n \in \mathbb{N}_{0} .
$$

Since the Biggins martingale is nonnegative, it converges a.s. to a finite limit $Z \geq 0$ with $\mathbb{E}[Z] \leq 1$. Further, it holds that $W=Z$ a. s. by Theorem 3.3 in [8].

What is more, if (A1) through (A4) hold, then

$$
\frac{m(2 \alpha)}{m(\alpha)^{2}}=m(2 \alpha)<1 \text {. }
$$

and in view of Theorem 1 in [3] or Theorem 2.1 in [20] it holds $Z_{n} \rightarrow Z$ in $L^{2}$. In particular, $\mathbb{E}[W]=\mathbb{E}[Z]=1$ and $\operatorname{Var}[W]=\operatorname{Var}[Z]=\mathbb{E}\left[(Z-1)^{2}\right]<\infty$. Using the fact that martingale increments are uncorrelated, we calculate $\operatorname{Var}[W]$ as follows:

$$
\begin{aligned}
\sigma_{W}^{2} & :=\operatorname{Var}[W]=\mathbb{E}\left[(Z-1)^{2}\right]=\sum_{n \geq 0} \mathbb{E}\left[\left(Z_{n+1}-Z_{n}\right)^{2}\right] \\
& =\sum_{n \geq 0} \mathbb{E}\left[\sum_{|u|=n} e^{-2 \alpha S(u)}\right] \mathbb{E}\left[\left(Z_{1}-1\right)^{2}\right]=\frac{\sigma^{2}}{1-m(2 \alpha)},
\end{aligned}
$$

where $\sigma^{2}=\mathbb{E}\left[\left(Z_{1}-1\right)^{2}\right]$.

\subsection{Fluctuations of Nerman's martingale}

Recall the notation $\mathcal{F}_{\infty}:=\sigma\left(\mathcal{F}_{n}: n \in \mathbb{N}_{0}\right)$ and denote by $\mathcal{L}(X)$ the law (distribution) of a random variable $X$. If $X, X_{t}, t \geq 0$ are real-valued random variables, we write

$$
\mathcal{L}\left(X_{t} \mid \mathcal{H}_{t}\right) \stackrel{\mathrm{w}}{\rightarrow} \mathcal{L}\left(X \mid \mathcal{F}_{\infty}\right) \quad \text { in } \mathbb{P} \text {-probability as } t \rightarrow \infty
$$

(in words, 'the distribution of $X_{t}$ given $\mathcal{H}_{t}$ converges weakly to the distribution of $X$ given $\mathcal{F}_{\infty}$ in $\mathbb{P}$-probability') if $\mathbb{E}\left[f\left(X_{t}\right) \mid \mathcal{H}_{t}\right] \stackrel{\mathbb{P}}{\rightarrow} \mathbb{E}\left[f(X) \mid \mathcal{F}_{\infty}\right]$ as $t \rightarrow \infty$ for every bounded 
continuous function $f: \mathbb{R} \rightarrow \mathbb{R}$. Notice that (2.11) implies $X_{t} \stackrel{d}{\rightarrow} X$ as $t \rightarrow \infty$, where $\stackrel{d}{\rightarrow}$ denotes convergence in distribution in $\mathbb{R}$.

Theorem 2.1 gives the asymptotic fluctuations of $\left(W_{t}\right)_{t \geq 0}$.

Theorem 2.1. Suppose that (A1) through (A4) hold. Then

$$
\mathcal{L}\left(e^{\alpha t / 2}\left(W-W_{t}\right) \mid \mathcal{H}_{t}\right) \stackrel{\mathrm{m}}{\rightarrow} \mathcal{L}\left(\left(\frac{\sigma^{2}}{-\alpha m^{\prime}(\alpha)} W\right)^{1 / 2} \cdot X \mid \mathcal{F}_{\infty}\right)
$$

in probability as $t \rightarrow \infty$ where $X$ is standard normal and independent of $\mathcal{F}_{\infty}$.

Convergence in distribution of $e^{\alpha t / 2}\left(W-W_{t}\right)$ as $t \rightarrow \infty$ can be strengthened to convergence in distribution of the stochastic process $\left(e^{\alpha t / 2}\left(W-W_{t+s}\right)\right)_{s \in \mathbb{R}}$ as $t \rightarrow \infty$ in the Skorokhod space $D(\mathbb{R})$, equipped with the $J_{1}$-topology (Chapter 12 in [5]). We write ' $\Rightarrow$ ' to denote convergence in distribution of random elements in this space.

Theorem 2.2. Suppose that (A1) through (A4) hold. Then

$$
\left(e^{\alpha t / 2}\left(W-W_{t+s}\right)\right)_{s \in \mathbb{R}} \Rightarrow\left(\left(\frac{\sigma^{2}}{-\alpha m^{\prime}(\alpha)} W\right)^{1 / 2} B_{e^{-\alpha s}}\right)_{s \in \mathbb{R}} \text { as } t \rightarrow \infty
$$

where $\left(B_{s}\right)_{s \geq 0}$ is a standard Brownian motion independent of $W$.

Finally, we deal with the almost sure fluctuations of Nerman's martingale, namely, we formulate a law of the iterated logarithm.

Theorem 2.3. If (A1) through (A5) hold, then, a. s. on the survival set $\mathcal{S}$,

$$
\begin{aligned}
& \limsup _{t \rightarrow \infty} \frac{e^{\alpha t / 2}}{\sqrt{\log t}}\left(W-W_{t}\right)=\left(\frac{2 \sigma^{2}}{-\alpha m^{\prime}(\alpha)} W\right)^{1 / 2}, \\
& \liminf _{t \rightarrow \infty} \frac{e^{\alpha t / 2}}{\sqrt{\log t}}\left(W-W_{t}\right)=-\left(\frac{2 \sigma^{2}}{-\alpha m^{\prime}(\alpha)} W\right)^{1 / 2} .
\end{aligned}
$$

Remark 2.4. It can be checked that $\mathbb{E}\left[\left(W-W_{t}\right)^{2}\right]=\operatorname{Var}[W] \mathbb{E}\left[\sum_{u \in \mathcal{I}_{t}} e^{-2 \alpha S(u)}\right]$. Hence, by Lemma 3.1 below, $e^{\alpha t} \mathbb{E}\left[\left(W-W_{t}\right)^{2}\right]$ converges as $t \rightarrow \infty$ to a positive constant. Thus, $\log t$ in (2.14) and (2.15) can be replaced by the asymptotically equivalent function $\log \left(\left|\log \mathbb{E}\left[\left(W-W_{t}\right)^{2}\right]\right|\right)$. This demonstrates that Theorem 2.3 is indeed a law of the iterated logarithm.

\section{Proofs of the main results}

We start this section with some basic notation and discussions.

\subsection{Preliminaries}

\section{The shift operators}

Suppose that $\psi$ is a function of $\left(\xi_{v}\right)_{v \in \mathcal{I}}$, all offspring point processes. For a given $u \in \mathcal{I}$, we write $[\psi]_{u}$ for the very same function but applied to $\left(\left(\xi_{u v}\right)_{v \in \mathcal{I}}\right)$. In other words, $[\cdot]_{u}$ is a shift operator that shifts the ancestor to $u$. For instance, we have $\left[Z_{1}\right]_{u}=\sum_{|v|=1} e^{-\alpha X_{v}(u)}$. Further, $[Z]_{u}=\lim _{n \rightarrow \infty}\left[Z_{n}\right]_{u}$ a.s. and $[W]_{u}=\lim _{t \rightarrow \infty}\left[W_{t}\right]_{u}$ a.s. are the limits of the shifted martingales. Here,

$$
\left[W_{t}\right]_{u}=\sum_{v \in\left[\mathcal{I}_{t}\right]_{u}} e^{-\alpha(S(u v)-S(u))}, \quad t \geq 0 .
$$

If $\psi$ is a function of a real variable $t$ and $\left(\xi_{v}\right)_{v \in \mathcal{I}}$, i.e., $\psi_{t}=f\left(t,\left(\xi_{v}\right)_{v \in \mathcal{I}}\right)$ for some function $f$, then we write $[\psi \cdot]_{u} \circ t$ for $f\left(t,\left(\xi_{u v}\right)_{v \in \mathcal{I}}\right)$. This is particularly useful when $t$ is replaced by a function of $S(u)$, for instance, in this notation, we have $[\psi \cdot]_{u} \circ(t-S(u))=$ $f\left(t-S(u),\left(\xi_{u v}\right)_{v \in \mathcal{I}}\right)$. 


\section{Crump-Mode-Jagers processes}

For a function $\psi$ as above we may now define $\mathcal{Z}_{t}^{\psi}$ the general branching process counted with a characteristic $\psi$ (or the Crump-Mode-Jagers processes) by setting

$$
\mathcal{Z}_{t}^{\psi}:=\sum_{u \in \mathcal{I}}[\psi \cdot]_{u} \circ(t-S(u))
$$

The classical Nerman's result states that, under suitable assumptions,

$$
\mathcal{Z}_{t}^{\psi} \rightarrow \frac{W}{-m^{\prime}(\alpha)} \times \int \mathbb{E}\left[\psi_{t}\right] e^{-\alpha t} \mathrm{~d} t \quad \text { a.s. }
$$

\section{Recursive decomposition}

With this notation, one deduces the following decomposition for $W_{t+r}$, valid for $t, r \geq 0$,

$$
W_{t+r}=\sum_{u \in \mathcal{I}_{t}} e^{-\alpha S(u)}\left[W_{r+t-.}\right]_{u} \circ S(u) .
$$

Passing to the limit as $r \rightarrow \infty$, we infer (after a careful inspection, see Section 14 in [4] or Lemma 4.2 in [1])

$$
W=\sum_{u \in \mathcal{I}_{t}} e^{-\alpha S(u)}[W]_{u} \quad \text { a.s. }
$$

if (A1) through (A3) hold and $\mathbb{E}\left[Z_{1} \log ^{+} Z_{1}\right]<\infty$.

\section{Nerman's martingale as an $L^{2}$-martingale}

If (A1) through (A4) hold, then, according to the discussion preceding (2.10), $W=Z \in L^{2}$. Further, $W_{t}=\mathbb{E}\left[W \mid \mathcal{H}_{t}\right]$ a.s. for all $t \geq 0$, i.e., $\left(W_{t}\right)_{t \geq 0}$ is an $L^{2}$-bounded martingale and hence convergent in $L^{2}$ (with limit $W$, of course). We write $v_{t}:=\operatorname{Var}\left[W_{t}\right]=\mathbb{E}\left[\left(W_{t}-1\right)^{2}\right]$ for the variance of $W_{t}, t \in \mathbb{R}$. Since $\left(\left(W_{t}-1\right)^{2}\right)_{t \geq 0}$ is a right-continuous submartingale, the function $t \mapsto v_{t}$ is nondecreasing and right-continuous. We now identify $v_{t}$ for some relevant values of $t$. Trivially, $v_{t}=0$ for $t<0$. Further, since $W_{0}=Z_{1}$, we have $v_{0}=\operatorname{Var}\left[Z_{1}\right]=\sigma^{2}$. From $W_{t} \rightarrow W$ in $L^{2}$ and (2.10) we finally deduce

$$
v_{\infty}:=\lim _{t \rightarrow \infty} \mathbb{E}\left[\left(W_{t}-1\right)^{2}\right]=\mathbb{E}\left[(W-1)^{2}\right]=\sigma_{W}^{2}=\frac{\sigma^{2}}{1-m(2 \alpha)} .
$$

Moreover, Doob's maximal $L^{2}$-inequality (with $p=2$ ) gives

$$
\mathbb{E}\left[\sup _{s \geq 0}\left(W_{s}-1\right)^{2}\right] \leq 4 \mathbb{E}\left[(W-1)^{2}\right]
$$

i.e., $M_{t}:=\sup _{0 \leq s \leq t}\left|W_{s}-1\right| \in L^{2}$.

\subsection{Fluctuations of Nerman's martingale: proofs}

We start with an auxiliary result derived from Nerman's law of large numbers for the general branching process.

Lemma 3.1. Suppose that (A1) through (A3) hold. Then

$$
e^{\alpha t} \sum_{u \in \mathcal{I}_{t}} e^{-2 \alpha S(u)} \stackrel{\mathbb{P}}{\rightarrow} \frac{1-m(2 \alpha)}{-\alpha m^{\prime}(\alpha)} W \quad \text { as } t \rightarrow \infty .
$$




\section{Gaussian fluctuations of Nerman's martingale}

More generally, if $f \in D(\mathbb{R})$ is a nonnegative bounded function, then, as $t \rightarrow \infty$,

$$
e^{\alpha t} \sum_{u \in \mathcal{I}_{t}} e^{-2 \alpha S(u)} f(t-S(u)) \stackrel{\mathbb{P}}{\rightarrow} \frac{W}{-m^{\prime}(\alpha)} \mathbb{E}\left[\sum_{k=1}^{N} e^{-2 \alpha X_{k}} \int_{0}^{X_{k}} e^{\alpha x} f\left(x-X_{k}\right) \mathrm{d} x\right] .
$$

The convergence in (3.7) (and thus also in (3.6)) holds in the stronger

- $L^{1}$ sense if $\mathbb{E}\left[Z_{1} \log ^{+} Z_{1}\right]<\infty$,

- almost sure sense if (A5) holds.

Proof. Define

$$
\phi(t):=e^{2 \alpha t} \sum_{k=1}^{N} e^{-2 \alpha X_{k}} \mathbb{1}_{\left[0, X_{k}\right)}(t) f\left(t-X_{k}\right), \quad t \in \mathbb{R}
$$

and notice that, for any $t \geq 0$, with $\|f\|_{\infty}:=\sup _{x \in \mathbb{R}} f(x)$,

$$
\mathbb{E}\left[\sup _{s \leq t} \phi(s)\right] \leq e^{2 \alpha t} \mathbb{E}\left[\sum_{k=1}^{N} e^{-2 \alpha X_{k}}\right]\|f\|_{\infty}=e^{2 \alpha t} m(2 \alpha)\|f\|_{\infty}<\infty,
$$

that is, Condition (3.2) in [22] holds. Further, for $t \geq 0$,

$$
\mathbb{E}\left[e^{-\alpha t} \phi(t)\right]=e^{\alpha t} \mathbb{E}\left[\sum_{k=1}^{N} e^{-2 \alpha X_{k}} \mathbb{1}_{\left[0, X_{k}\right)}(t) f\left(t-X_{k}\right)\right]=e^{\alpha t} \int_{(t, \infty)} e^{-2 \alpha x} f(t-x) \mu(\mathrm{d} x)
$$

is càdlàg as a function of $t$ by the dominated convergence theorem. On the other hand, the inequality

$$
\begin{aligned}
\mathbb{E}\left[e^{-\alpha t} \phi(t)\right] & =e^{\alpha t} \mathbb{E}\left[\sum_{k=1}^{N} e^{-2 \alpha X_{k}} \mathbb{1}_{\left\{X_{k}>t\right\}} f\left(t-X_{k}\right)\right] \\
& \leq\|f\|_{\infty} \mathbb{E}\left[\sum_{k=1}^{N} e^{-\alpha X_{k}} \mathbb{1}_{\left\{X_{k}>t\right\}}\right]=\|f\|_{\infty} \int_{(t, \infty)} e^{-\alpha x} \mu(\mathrm{d} x)
\end{aligned}
$$

together with (2.4) shows that the nonnegative càdlàg function $t \mapsto \mathbb{E}\left[e^{-\alpha t} \phi(t)\right]$ is bounded from above by a directly Riemann integrable function on $[0, \infty)$, hence it is directly Riemann integrable on $[0, \infty)$. Consequently, the assumptions of Theorem 3.1 in [22] are satisfied. The cited theorem gives that, as $t \rightarrow \infty$,

$$
\begin{aligned}
e^{\alpha t} \sum_{u \in \mathcal{I}_{t}} e^{-2 \alpha S(u)} f(t-S(u)) & =e^{-\alpha t} \sum_{u \in \mathcal{G}}[\phi]_{u}(t-S(u))=e^{-\alpha t} \mathcal{Z}_{t}^{\phi} \\
& \rightarrow \frac{W}{-m^{\prime}(\alpha)} \mathbb{E}\left[\sum_{k=1}^{N} e^{-2 \alpha X_{k}} \int_{0}^{X_{k}} e^{\alpha x} f\left(x-X_{k}\right) \mathrm{d} x\right] .
\end{aligned}
$$

In the special case $f=1$, we find

$$
e^{\alpha t} \sum_{u \in \mathcal{I}_{t}} e^{-2 \alpha S(u)} \stackrel{\mathbb{P}}{\rightarrow} \frac{W}{-m^{\prime}(\alpha)} \mathbb{E}\left[\sum_{k=1}^{N} e^{-2 \alpha X_{k}} \int_{0}^{X_{k}} e^{\alpha x} \mathrm{~d} x\right]=\frac{1-m(2 \alpha)}{-\alpha m^{\prime}(\alpha)} W .
$$

As for the $L^{1}$-convergence, use Corollary 3.3 in [22].

Finally, we pass to the a.s. convergence. Since $\phi$ is càdlàg and

$$
e^{-\alpha t} \phi(t)=e^{\alpha t} \sum_{k=1}^{N} e^{-2 \alpha X_{k}} \mathbb{1}_{\left[0, X_{k}\right)}(t) f\left(t-X_{k}\right) \leq\|f\|_{\infty} \sum_{k=1}^{N} e^{-\alpha X_{k}} \mathbb{1}_{\left[0, X_{k}\right)}(t),
$$




\section{Gaussian fluctuations of Nerman's martingale}

condition (2.6), which is Condition 5.1 from [22], entails

$$
\mathbb{E}\left[\sup _{t \geq 0} \frac{e^{-\alpha t} \phi(t)}{g(t)}\right]<\infty
$$

This is Condition 5.2 from [22] with the particular $\phi$ and $h=g$. Thus, the assumptions of Theorem 5.4 in [22] are satisfied. According to this theorem, the convergence in (3.6) holds also in the almost sure sense.

The lemma has the following corollary, which we use in the proofs of Theorems 2.2 and 2.3, but not in the proof of Theorem 2.1.

Corollary 3.2. Suppose that (A1) through (A4) hold. Then, for any fixed $\delta>0$, as $t \rightarrow \infty$,

$$
e^{\alpha t} \sum_{u \in \mathcal{I}_{t}} e^{-2 \alpha S(u)} v_{\delta+t-S(u)} \rightarrow c_{\delta} W \quad \text { in } L^{1}
$$

where

$$
c_{\delta}:=\frac{1}{-m^{\prime}(\alpha)} \mathbb{E}\left[\sum_{k=1}^{N} e^{-2 \alpha X_{k}} \int_{0}^{X_{k}} e^{\alpha x} v_{\delta+x-X_{k}} \mathrm{~d} x\right] .
$$

The convergence in (3.11) holds in the stronger almost sure sense if (A5) holds. Further, $c_{\delta}$ is nondecreasing as a function of $\delta$ with $c_{\delta}>0$ for every $\delta>0$. The limits of $c_{\delta}$ as $\delta \rightarrow 0$ and $\delta \rightarrow \infty$ are given by

$$
\lim _{\delta \downarrow 0} c_{\delta}=0 \quad \text { and } \quad c_{\infty}:=\lim _{\delta \uparrow \infty} c_{\delta}=\frac{\sigma^{2}}{-\alpha m^{\prime}(\alpha)} .
$$

Proof. The validity of (A4) implies that the function $t \mapsto v_{t}$ is bounded by (3.4). From the discussion preceding (3.4), we infer that $t \mapsto v_{t}$ is nondecreasing and right-continuous, hence càdlàg. Thus, for any fixed $\delta>0$, (3.11) follows from Lemma 3.1 with $f(t)=v_{\delta+t}$, $t \in \mathbb{R}$. We infer $c_{\delta}>0$ from $0<\sigma^{2}=v_{0} \leq v_{t}$ for $t \geq 0$ and the representation

$$
\int_{0}^{X_{k}} e^{\alpha x} v_{\delta+x-X_{k}} \mathrm{~d} x=\mathbb{1}_{\left\{X_{k} \leq \delta\right\}} \int_{0}^{X_{k}} e^{\alpha x} v_{\delta+x-X_{k}} \mathrm{~d} x+\mathbb{1}_{\left\{X_{k}>\delta\right\}} \int_{X_{k}-\delta}^{X_{k}} e^{\alpha x} v_{\delta+x-X_{k}} \mathrm{~d} x>0 .
$$

Since $t \mapsto v_{t}$ is a nondecreasing function, so is $\delta \mapsto c_{\delta}$. Hence, $\lim _{\delta \downarrow 0} c_{\delta}$ and $c_{\infty}:=$ $\lim _{\delta \uparrow \infty} c_{\delta}$ exist. Since $v_{t}=0$ for $t<0$ and $\lim _{t \rightarrow \infty} v_{t}=\sigma^{2} /(1-m(2 \alpha))$, (3.13) follows with the help of the monotone convergence theorem.

Proof of Theorem 2.1. For $t \geq 0$, we infer from (3.3)

$$
e^{\alpha t / 2}\left(W-W_{t}\right)=e^{\alpha t / 2} \sum_{u \in \mathcal{I}_{t}} e^{-\alpha S(u)}\left([W]_{u}-1\right) \quad \text { a.s. },
$$

which given $\mathcal{H}_{t}$ is a weighted sum of independent, centered and square-integrable random variables (Theorem 4.14 in [18]). We show that the distribution of this sum given $\mathcal{H}_{t}$ converges in probability to the distribution of a centered normal random variable. For simplicity, we write $\mathbb{E}_{t}[\cdot]$ to denote the conditional expectation given $\mathcal{H}_{t}$. Then, by (2.10),

$$
\begin{aligned}
\mathbb{E}_{t}\left[\left(e^{\alpha t / 2} \sum_{u \in \mathcal{I}_{t}} e^{-\alpha S(u)}\left([W]_{u}-1\right)\right)^{2}\right] & =e^{\alpha t} \mathbb{E}_{t}\left[\sum_{u \in \mathcal{I}_{t}} e^{-2 \alpha S(u)}\left([W]_{u}-1\right)^{2}\right] \\
& =\frac{\sigma^{2}}{1-m(2 \alpha)} e^{\alpha t} \sum_{u \in \mathcal{I}_{t}} e^{-2 \alpha S(u)} .
\end{aligned}
$$




\section{Gaussian fluctuations of Nerman's martingale}

Observe that $\mathbb{E}\left[Z_{1}^{2}\right]<\infty$ entails $\mathbb{E}\left[Z_{1} \log ^{+} Z_{1}\right]<\infty$ whence, by Lemma 3.1, (3.6) holds in $L^{1}$ and thereupon

$$
\mathbb{E}_{t}\left[\left(e^{\alpha t / 2} \sum_{u \in \mathcal{I}_{t}} e^{-\alpha S(u)}\left([W]_{u}-1\right)\right)^{2}\right] \rightarrow \frac{\sigma^{2}}{-\alpha m^{\prime}(\alpha)} W \quad \text { in } L^{1}
$$

as $t \rightarrow \infty$. Further, for $x \geq 0$, we define

$$
\sigma_{W}^{2}(x):=\mathbb{E}\left[(W-1)^{2} \mathbb{1}_{\{|W-1|>x\}}\right]
$$

and notice that $\lim _{x \rightarrow \infty} \sigma_{W}^{2}(x)=0$ by (3.4) and the dominated convergence theorem. Consequently, for all $\varepsilon>0$,

$$
\begin{aligned}
\sum_{u \in \mathcal{I}_{t}} \mathbb{E}_{t}\left[\left(e^{\alpha t / 2} e^{-\alpha S(u)}\left([W]_{u}-1\right)\right)^{2} \mathbb{1}_{\left\{\left|e^{\alpha t / 2} e^{-\alpha S(u)}\left([W]_{u}-1\right)\right|>\varepsilon\right\}}\right] \\
=e^{\alpha t} \sum_{u \in \mathcal{I}_{t}} e^{-2 \alpha S(u)} \sigma_{W}^{2}\left(\varepsilon e^{-\alpha t / 2} e^{\alpha S(u)}\right) \leq \sigma_{W}^{2}\left(\varepsilon e^{\alpha t / 2}\right) e^{\alpha t} \sum_{u \in \mathcal{I}_{t}} e^{-2 \alpha S(u)} \stackrel{\mathbb{P}}{\rightarrow} 0
\end{aligned}
$$

as $t \rightarrow \infty$ by (3.6). Thus, (2.5) through (2.7) in [9] hold, and we conclude (2.12).

For the proof of the functional central limit theorem, Theorem 2.2, we need some preparatory lemmas.

Lemma 3.3. Suppose that (A1) through (A4) hold. Then the family $\left(\left(W-W_{t}\right)^{2}\right)_{t \in \mathbb{R}}$ is uniformly integrable. In other words, the function $\sigma_{t}^{2}(x):=\mathbb{E}\left[\left|W-W_{t}\right|^{2} \mathbb{1}_{\left\{\left|W-W_{t}\right|>x\right\}}\right]$, $t \in \mathbb{R}, x \geq 0$ is a bounded on $\mathbb{R} \times[0, \infty)$ with

$$
\sup _{t \in \mathbb{R}} \sigma_{t}^{2}(x) \rightarrow 0 \quad \text { as } x \rightarrow \infty .
$$

Proof. From the discussion preceding (3.4), we know that $W_{t} \rightarrow W$ a.s. and in $L^{2}$, and that $W_{t}=\mathbb{E}\left[W \mid \mathcal{H}_{t}\right]$ a. s. Thus, the family $\left(W_{t}^{2}\right)_{t \geq 0}$ is uniformly integrable, hence so is the family $\left(\left(W-W_{t}\right)^{2}\right)_{t \geq 0}$, which implies (3.16).

Lemma 3.4. Suppose that (A1) through (A4) hold and let $0 \leq r<s<\infty$. Then, as $t \rightarrow \infty$ and with $\operatorname{Cov}_{t}[\cdot, \cdot]$ and $\operatorname{Var}_{t}[\cdot]$ denoting conditional covariance and variance given $\mathcal{H}_{t}$, respectively,

$$
\begin{aligned}
\operatorname{Cov}_{t}\left[e^{\alpha t / 2}\left(W-W_{t+r}\right), e^{\alpha t / 2}\left(W-W_{t+s}\right)\right] & =\operatorname{Var}_{t}\left[e^{\alpha t / 2}\left(W-W_{t+s}\right)\right] \\
& \rightarrow \frac{e^{-\alpha s} \sigma^{2}}{-\alpha m^{\prime}(\alpha)} W \quad \text { in } L^{1}
\end{aligned}
$$

Notice that the expression on the right-hand side of (3.17) is exactly the (conditional) covariance of the limiting process in (2.13).

Proof. For any $t \geq 0$, we have

$$
\begin{aligned}
\operatorname{Cov}_{t} & {\left[e^{\alpha t / 2}\left(W-W_{t+r}\right), e^{\alpha t / 2}\left(W-W_{t+s}\right)\right] } \\
& =\operatorname{Cov}_{t}\left[e^{\alpha t / 2}\left(W-W_{t+s}+W_{t+s}-W_{t+r}\right), e^{\alpha t / 2}\left(W-W_{t+s}\right)\right] \\
& =\operatorname{Var}_{t}\left[e^{\alpha t / 2}\left(W-W_{t+s}\right)\right]+\operatorname{Cov}_{t}\left[e^{\alpha t / 2}\left(W_{t+s}-W_{t+r}\right), e^{\alpha t / 2}\left(W-W_{t+s}\right)\right] \\
& =\operatorname{Var}_{t}\left[e^{\alpha t / 2}\left(W-W_{t+s}\right)\right] .
\end{aligned}
$$




\section{Gaussian fluctuations of Nerman's martingale}

since increments of square-integrable martingales are (conditionally) uncorrelated. It thus remains to investigate $\operatorname{Var}_{t}\left[e^{\alpha t / 2}\left(W-W_{t+s}\right)\right]$ as $t \rightarrow \infty$. Here, arguing as in the proof of Theorem 2.1 and using Lemma 3.1, we infer, with $\sigma_{t}^{2}=\mathbb{E}\left[\left(W-W_{t}\right)^{2}\right]$ for $t \in \mathbb{R}$,

$$
\begin{aligned}
\operatorname{Var}_{t}\left[e^{\alpha t / 2}\left(W-W_{t+s}\right)\right] & =e^{\alpha t} \sum_{u \in \mathcal{I}_{t}} e^{-2 \alpha S(u)} \sigma_{t+s-S(u)}^{2} \\
& \rightarrow \frac{W}{-m^{\prime}(\alpha)} \mathbb{E}\left[\sum_{k=1}^{N} e^{-2 \alpha X_{k}} \int_{0}^{X_{k}} e^{\alpha x} \sigma_{s+x-X_{k}}^{2} \mathrm{~d} x\right]=: d_{s} W
\end{aligned}
$$

as $t \rightarrow \infty$ in $L^{1}$, where $d_{s} \geq 0$ is a constant. We now calculate the constant $d_{s}$, but avoid evaluating it directly. Instead, notice that

$$
\begin{aligned}
\operatorname{Var}_{t}\left[e^{\alpha t / 2}\left(W-W_{t+s}\right)\right] & =\mathbb{E}_{t}\left[\left(e^{\alpha t / 2}\left(W-W_{t+s}\right)\right)^{2}\right] \\
& =\mathbb{E}_{t}\left[\mathbb{E}_{t+s}\left[\left(e^{\alpha t / 2}\left(W-W_{t+s}\right)\right)^{2}\right]\right]
\end{aligned}
$$

By (3.15), $\mathbb{E}_{t+s}\left[\left(e^{\alpha t / 2}\left(W-W_{t+s}\right)\right)^{2}\right]$ converges in $L^{1}$ as $t \rightarrow \infty$, in particular, the family $\left(\mathbb{E}_{t+s}\left[\left(e^{\alpha t / 2}\left(W-W_{t+s}\right)\right)^{2}\right]\right)_{t \geq 0}$ is uniformly integrable. Hence so is $\left(\mathbb{E}_{t}\left[\left(e^{\alpha t / 2}(W-\right.\right.\right.$ $\left.\left.\left.\left.W_{t+s}\right)\right)^{2}\right]\right)_{t \geq 0}$. Consequently,

$$
\mathbb{E}\left[\mathbb{E}_{t}\left[\left(e^{\alpha t / 2}\left(W-W_{t+s}\right)\right)^{2}\right]\right] \rightarrow d_{s} \text { as } t \rightarrow \infty
$$

On the other hand,

$$
\mathbb{E}\left[\mathbb{E}_{t}\left[\left(e^{\alpha t / 2}\left(W-W_{t+s}\right)\right)^{2}\right]\right]=e^{-\alpha s} \mathbb{E}\left[\mathbb{E}_{t}\left[\left(e^{\alpha(t+s) / 2}\left(W-W_{t+s}\right)\right)^{2}\right]\right] \rightarrow \frac{e^{-\alpha s} \sigma^{2}}{-\alpha m^{\prime}(\alpha)}
$$

by (3.15). Hence, $d_{s}=\frac{e^{-\alpha s} \sigma^{2}}{-\alpha m^{\prime}(\alpha)}$.

Proof of Theorem 2.2. We first prove weak convergence of the finite-dimensional distributions on $[0, \infty)$. To this end, fix $n \in \mathbb{N}$ and $0 \leq s_{1}<s_{2} \ldots<s_{n}$. Abbreviate $t+s_{k}$ by $t_{k}, k=1, \ldots, n$ and define $t_{n+1}:=\infty$ and $W_{t_{n+1}}:=W$. We use the Cramér-Wold device which reduces the problem to studying the convergence in law of the following linear combinations:

$$
\sum_{k=1}^{n} \gamma_{k} e^{\alpha t / 2}\left(W-W_{t_{k}}\right)
$$

for fixed $\gamma_{1}, \ldots, \gamma_{n} \in \mathbb{R}$. Recall that $\operatorname{Var}_{t}$ and $\operatorname{Cov}_{t}$ denote the conditional variance and covariance given $\mathcal{H}_{t}$, respectively. Lemma 3.4 yields

$$
\begin{aligned}
\operatorname{Var}_{t} & {\left[\sum_{k=1}^{n} \gamma_{k} e^{\alpha t / 2}\left(W-W_{t_{k}}\right)\right] } \\
& =\sum_{k=1}^{n} \gamma_{k}^{2} e^{\alpha t} \operatorname{Var}_{t}\left[W-W_{t_{k}}\right]+2 \sum_{1 \leq j<k \leq n} \gamma_{j} \gamma_{k} \operatorname{Cov}_{t}\left[W-W_{t_{j}}, W-W_{t_{k}}\right] \\
& \rightarrow\left(\sum_{k=1}^{n} \gamma_{k}^{2} e^{-\alpha s_{k}}+2 \sum_{1 \leq j<k \leq n} \gamma_{j} \gamma_{k} e^{-\alpha s_{k}}\right) \frac{\sigma^{2}}{-\alpha m^{\prime}(\alpha)} W \text { in } L^{1}
\end{aligned}
$$

as $t \rightarrow \infty$. Note that the expression in the last line is exactly the conditional variance of the linear combinations corresponding to the limiting process in (2.13) since

$$
\begin{gathered}
\operatorname{Var}\left[\sum_{k=1}^{n} \gamma_{k}\left(\frac{\sigma^{2}}{-\alpha m^{\prime}(\alpha)} W\right)^{1 / 2} B_{e^{-\alpha s_{k}}} \mid W\right]=\operatorname{Var}\left[\sum_{k=1}^{n} \gamma_{k} B_{e^{-\alpha s_{k}}}\right] \frac{\sigma^{2}}{-\alpha m^{\prime}(\alpha)} W \\
=\left(\sum_{k=1}^{n} \gamma_{k}^{2} e^{-\alpha s_{k}}+2 \sum_{1 \leq j<k \leq n} \gamma_{j} \gamma_{k} e^{-\alpha s_{k}}\right) \frac{\sigma^{2}}{-\alpha m^{\prime}(\alpha)} W \text { a.s. }
\end{gathered}
$$




\section{Gaussian fluctuations of Nerman's martingale}

Next, we check the Lindeberg-Feller condition. Using the decomposition

$$
\sum_{k=1}^{n} \gamma_{k} e^{\alpha t / 2}\left(W-W_{t_{k}}\right)=\sum_{k=1}^{n} \gamma_{k} e^{\alpha t / 2} \sum_{u \in \mathcal{I}_{t}} e^{-\alpha S(u)}\left([W]_{u}-\left[W_{t_{k}-.}\right]_{u} \circ(S(u))\right),
$$

we argue as in the proof of Theorem 2.1. Recalling the notation $\sigma_{r}^{2}(x)=\mathbb{E}[\mid W-$ $\left.\left.W_{r}\right|^{2} \mathbb{1}_{\left\{\left|W-W_{r}\right|>x\right\}}\right\}$, we infer, for all $\varepsilon>0$,

$$
\begin{aligned}
\sum_{u \in \mathcal{I}_{t}} \mathbb{E}_{t}\left[\left(e^{\alpha(t / 2-S(u))}\left([W]_{u}-\left[W_{t_{k}-} \cdot\right]_{u} \circ(S(u))\right)\right)^{2}\right. \\
\left.\quad \cdot \mathbb{1}_{\left\{\left|e^{\alpha(t / 2-S(u))}\left([W]_{u}-\left[W_{t_{k}-} \cdot\right]_{u} \circ(S(u))\right)\right|>\varepsilon\right\}}\right] \\
=e^{\alpha t} \sum_{u \in \mathcal{I}_{t}} e^{-2 \alpha S(u)} \sigma_{t_{k}-S(u)}^{2}\left(e^{-\alpha t / 2} e^{\alpha S(u)} \varepsilon\right) \stackrel{P}{\rightarrow} 0
\end{aligned}
$$

by (3.6) and (3.16).

This implies that the finite-dimensional distributions of $\left(e^{\alpha t / 2}\left(W-W_{t+s}\right)\right)_{s \geq 0}$ given $\mathcal{H}_{t}$ converge weakly to those of $\left(\left(\sigma^{2} /\left(-\alpha m^{\prime}(\alpha)\right) W\right)^{1 / 2} B_{e^{-\alpha s}}\right)_{s \geq 0}$ given $\mathcal{F}_{\infty}$. From this, we conclude that

$$
\left(e^{\alpha t / 2}\left(W-W_{t+s}\right)\right)_{s \geq 0} \stackrel{\text { fdd }}{\longrightarrow}\left(\left(\frac{\sigma^{2}}{-\alpha m^{\prime}(\alpha)} W\right)^{1 / 2} B_{e^{-\alpha s}}\right)_{s \geq 0} \text { as } t \rightarrow \infty
$$

where $\stackrel{\text { fdd }}{\longrightarrow}$ denotes weak convergence of finite-dimensional distributions.

The next step is to check that the distributions of the family

$$
\left(e^{\alpha t / 2}\left(W_{t}-W_{t+s}\right)\right)_{s \geq 0}, t \geq 0 \text { are tight in } D([0, \infty)) .
$$

We use Aldous's tightness criterion, see e.g. Theorem 16.10 on p. 178 of [5]. To this end, we first check condition (16.22) in the cited source, i.e.,

$$
\lim _{x \rightarrow \infty} \limsup _{t \rightarrow \infty} \mathbb{P}\left(\sup _{0 \leq s \leq b}\left|e^{\alpha t / 2}\left(W_{t}-W_{t+s}\right)\right| \geq x\right)=0 \quad \text { for all } b>0 .
$$

For any fixed $b, t, x>0$, by Chebyshev's inequality and Doob's maximal $L^{p}$-inequality (with $p=2$ ),

$$
\mathbb{P}\left(\sup _{0 \leq s \leq b}\left|e^{\alpha t / 2}\left(W_{t}-W_{t+s}\right)\right| \geq x\right) \leq \frac{4 e^{\alpha t}}{x^{2}} \mathbb{E}\left[\left|W_{t+b}-W_{t}\right|^{2}\right] \quad \text { for all } b>0 .
$$

Here, an application of (3.11) yields

$$
\begin{aligned}
e^{\alpha t} \mathbb{E}\left[\left|W_{t+b}-W_{t}\right|^{2}\right] & =e^{\alpha t} \mathbb{E}\left[\left(\sum_{u \in \mathcal{I}_{t}} e^{-\alpha S(u)}\left(\left[W_{t+b-.}\right]_{u} \circ S(u)-1\right)\right)^{2}\right] \\
& =e^{\alpha t} \mathbb{E}\left[\sum_{u \in \mathcal{I}_{t}} e^{-2 \alpha S(u)} v_{t+b-S(u)}\right] \rightarrow c_{b} \quad \text { as } t \rightarrow \infty .
\end{aligned}
$$

Using this after taking the limsup as $t \rightarrow \infty$ in (3.22), and then letting $x \rightarrow \infty$ gives (3.21).

We now turn to the second condition of Aldous's criterion, namely, for all $\varepsilon, b>0$

$$
\lim _{\delta \rightarrow 0} \limsup _{t \rightarrow \infty} \sup _{\tau} \mathbb{P}\left(e^{\alpha t / 2}\left|\left(W_{t}-W_{t+\tau+\delta}\right)-\left(W_{t}-W_{t+\tau}\right)\right| \geq \varepsilon\right)=0
$$

where $\sup _{\tau}$ is the supremum over all discrete stopping times $0 \leq \tau \leq b$ with respect to the filtration $\left(\mathcal{H}_{t+s}\right)_{s \geq 0}$. Here, 'discrete' means that $\tau$ takes only finitely many values. To 


\section{Gaussian fluctuations of Nerman's martingale}

prove (3.23), fix $\varepsilon, b>0$ and let $\tau \leq b$ be a discrete stopping time. Then $\tau_{t}:=t+\tau$ is a stopping time with respect to $\left(\mathcal{H}_{s}\right)_{s \geq 0}$. We claim that $\mathcal{I}_{\tau_{t}}$ is an optional line. Clearly, it is a random line. Further, if $t \leq t_{1} \leq \ldots \leq t_{m} \leq t+b$ are the values $\tau$ takes, then, for any deterministic line $L \subseteq \mathcal{I}$,

$$
\left\{\mathcal{I}_{\tau_{t}} \preceq L\right\}=\bigcup_{k=1}^{m}\left(\left\{\tau_{t}=t_{k}\right\} \cap\left\{\mathcal{I}_{t_{k}} \preceq L\right\}\right) \in \mathcal{F}_{L}
$$

since $\tau_{t}$ is a stopping time with respect to $\left(\mathcal{H}_{s}\right)_{s \geq 0}$, thus $\left\{\tau_{t}=t_{k}\right\} \in \mathcal{H}_{t_{k}}=\mathcal{F}_{\mathcal{I}_{t_{k}}}$ and, consequently, $\left\{\tau_{t}=t_{k}\right\} \cap\left\{\mathcal{I}_{t_{k}} \preceq L\right\} \in \mathcal{F}_{L}$ by the definition of $\mathcal{F}_{\mathcal{I}_{t_{k}}}$. We may now use the strong Markov branching property (Theorem 4.14 in [18]) to conclude that

$$
\begin{aligned}
& \mathbb{P}\left(e^{\alpha t / 2}\left|\left(W_{t}-W_{t+\tau+\delta}\right)-\left(W_{t}-W_{t+\tau}\right)\right| \geq \varepsilon\right)=\mathbb{P}\left(e^{\alpha t / 2}\left|\left(W_{t+\tau+\delta}\right)-W_{t+\tau}\right| \geq \varepsilon\right) \\
& \quad \leq \frac{e^{\alpha t}}{\varepsilon^{2}} \mathbb{E}\left[\left|\left(W_{t+\tau+\delta}\right)-W_{t+\tau}\right|^{2}\right] \\
& \quad=\frac{e^{\alpha t}}{\varepsilon^{2}} \mathbb{E}\left[\mathbb{E}\left[\left(\sum_{u \in \mathcal{I}_{\tau_{t}}} e^{-\alpha S(u)}\left(\left[W_{\tau_{t}+\delta-.}\right]_{u} \circ S(u)-1\right)\right)^{2} \mid \mathcal{F}_{\mathcal{I}_{\tau_{t}}}\right]\right] \\
& \quad=\frac{e^{\alpha t}}{\varepsilon^{2}} \mathbb{E}\left[\sum_{u \in \mathcal{I}_{t+\tau}} e^{-2 \alpha S(u)} v_{t+\tau+\delta-S(u)}\right] .
\end{aligned}
$$

Here, if $0 \leq s_{1} \leq \ldots \leq s_{m} \leq b$ denote the values of $\tau$, then

$$
\begin{aligned}
e^{\alpha t} \sum_{u \in \mathcal{I}_{t+\tau}} e^{-2 \alpha S(u)} v_{t+\tau+\delta-S(u)} & \\
& =\sum_{k=1}^{m} e^{\alpha s_{k}} \mathbb{1}_{\left\{\tau=s_{k}\right\}} e^{-\alpha\left(t+s_{k}\right)} \sum_{u \in \mathcal{I}_{t+s_{k}}} e^{-2 \alpha S(u)} v_{t+\tau+\delta-S(u)} \\
& \rightarrow \sum_{k=1}^{m} e^{\alpha s_{k}} \mathbb{1}_{\left\{\tau=s_{k}\right\}} c_{\delta} W=e^{\alpha \tau} c_{\delta} W \quad \text { in } L^{1} \text { as } t \rightarrow \infty
\end{aligned}
$$

by Corollary 3.2. Hence,

$$
\limsup _{t \rightarrow \infty} \sup _{\tau} \mathbb{P}\left(e^{\alpha t / 2}\left|\left(W_{t}-W_{t+\tau+\delta}\right)-\left(W_{t}-W_{t+\tau}\right)\right| \geq \varepsilon\right) \leq \frac{1}{\varepsilon^{2}} e^{\alpha b} c_{\delta}
$$

Thus, (3.23) follows from (3.13). Combining (3.21) and (3.23) with Theorem 16.10 on p. 178 of [5] yields (3.20). Since the increments of the processes $\left(e^{\alpha t / 2}\left(W_{t}-W_{t+s}\right)\right)_{s \geq 0}$ and $\left(e^{\alpha t / 2}\left(W-W_{t+s}\right)\right)_{s \geq 0}$ are the same, Theorem 16.5 in [5] in combination with the fact that $e^{\alpha t / 2}\left(W-W_{t}\right)$ converges in distribution implies that also the distributions of the family $\left(e^{\alpha t / 2}\left(W-W_{t+s}\right)\right)_{s \geq 0}, t \geq 0$ are tight in $D([0, \infty))$. Together with the convergence of the finite-dimensional distributions, we obtain

$$
\left(e^{\alpha t / 2}\left(W-W_{t+s}\right)\right)_{s \geq 0} \Rightarrow\left(\left(\frac{\sigma^{2}}{-\alpha m^{\prime}(\alpha)} W\right)^{1 / 2} B_{e^{-\alpha s}}\right)_{s \geq 0} \text { as } t \rightarrow \infty \text { on } D([0, \infty)) .
$$

For any $r>0$ the shift operator $\theta_{r}: f(\cdot) \mapsto f(\cdot+r)$ is an isometry between $D([0, b])$ and $D([-r, b-r])$. Hence, $\theta_{r}$ is a continuous mapping from $D([0, \infty))$ to $D([-r, \infty))$. In particular, for any $r>0$, by the scaling invariance of Brownian motion, we infer

$$
\begin{aligned}
\left(e^{\alpha t / 2}\left(W-W_{t+s}\right)\right)_{s \geq-r} & =\theta_{r}\left(\left(e^{\alpha t / 2}\left(W-W_{t-r+s}\right)\right)\right)_{s \geq-r} \\
& \Rightarrow \theta_{r}\left(e^{\alpha r / 2}\left(\frac{\sigma^{2}}{-\alpha m^{\prime}(\alpha)} W\right)^{1 / 2} B_{e^{-\alpha s}}\right)_{s \geq-r} \\
& \stackrel{\text { law }}{=}\left(\left(\frac{\sigma^{2}}{-\alpha m^{\prime}(\alpha)} W\right)^{1 / 2} B_{e^{-\alpha s}}\right)_{s \geq 0},
\end{aligned}
$$


as $t \rightarrow \infty$ on $D([-r, \infty))$. Here, $\stackrel{\text { law }}{=}$ denotes equality of distributions. Since this holds for every $r>0$, we arrive at (2.13).

\subsection{Law of the iterated logarithm: proofs}

The key tool in our proof of the law of the iterated logarithm for Nerman's martingale is Proposition 7.2 on p. 436 in [2].

Lemma 3.5. Let $\left(\mathcal{R}_{n}\right)_{n \in \mathbb{N}_{0}}$ be an increasing sequence of $\sigma$-fields and $\left(T_{n}\right)_{n \in \mathbb{N}_{0}}$ be a sequence of random variables such that

$$
\sum_{n \geq 0} \sup _{y \in \mathbb{R}}\left|\mathbb{P}\left(T_{n} \leq y \mid \mathcal{R}_{n}\right)-\Phi(y)\right|<\infty \quad \text { a.s. }
$$

where $\Phi(y):=\frac{1}{\sqrt{2 \pi}} \int_{-\infty}^{y} e^{-x^{2} / 2} \mathrm{~d} x, y \in \mathbb{R}$. Then

$$
\limsup _{n \rightarrow \infty} \frac{T_{n}}{\sqrt{\log n}} \leq \sqrt{2} \text { a.s. }
$$

If there is a $k \in \mathbb{N}$ such that $T_{n}$ is $\mathcal{R}_{n+k}$-measurable for each $n \in \mathbb{N}_{0}$, then

$$
\limsup _{n \rightarrow \infty} \frac{T_{n}}{\sqrt{\log n}}=\sqrt{2} \text { a.s. }
$$

The next result is Lemma A.2 in [13], an infinite version of the Berry-Esseen inequality for independent, centered random variables. It is likely that this fact is also given in other sources.

Lemma 3.6. Let $Y_{1}, Y_{2}, \ldots$ be independent random variables with $\mathbb{E}\left[Y_{i}\right]=0, \sigma_{Y_{i}}^{2}:=$ $\operatorname{Var}\left[Y_{i}\right]<\infty$ and $\rho_{Y_{i}}:=\mathbb{E}\left[\left|Y_{i}\right|^{3}\right], i \in \mathbb{N}$. If $\sum_{i \geq 1} \sigma_{Y_{i}}^{2}<\infty$, then, for an absolute constant $C$,

$$
\sup _{y \in \mathbb{R}}\left|\mathbb{P}\left(\frac{\sum_{i \geq 1} Y_{i}}{\left(\sum_{i \geq 1} \sigma_{Y_{i}}^{2}\right)^{1 / 2}} \leq y\right)-\Phi(y)\right| \leq C \frac{\sum_{i \geq 1} \rho_{Y_{i}}}{\left(\sum_{i \geq 1} \sigma_{Y_{i}}^{2}\right)^{3 / 2}}
$$

Recall that the limits in (2.14) and (2.15) are considered on the survival set $\mathcal{S}$. We only give a complete proof for the upper limit. Investigating $W_{t}-W$ rather than $W-W_{t}$ gives the result for the lower limit at no extra cost. Although the scheme of the proof is similar to that of Theorem 3.4 on p. 130 in [2] in which a Markov branching process was investigated, technical details differ at places. Without loss of generality we assume in what follows that $\mathbb{P}(\mathcal{S})=1$ (otherwise we have to use Lemma 3.5 with the probability measure $\mathbb{P}$ replaced with $\mathbb{P}(\cdot \mid \mathcal{S})$ and write "a. s. on the survival set $\mathcal{S}$ " rather than "a. s." throughout). This assumption ensures that $W$ is positive a.s. rather than with positive probability.

For $t, r>0$, we use the following representations derived from (3.2) and (3.3):

$$
\begin{aligned}
W_{t+r}-W_{t} & =\sum_{u \in \mathcal{I}_{t}} e^{-\alpha S(u)}\left(\left[W_{r+t-.}\right]_{u} \circ S(u)-1\right) \\
\text { and } W-W_{t} & =\sum_{u \in \mathcal{I}_{t}} e^{-\alpha S(u)}\left([W]_{u}-1\right) .
\end{aligned}
$$

Recall that the $S(u), u \in \mathcal{I}_{t}$ are $\mathcal{H}_{t}$-measurable, whereas the $\left[W_{r-x}\right]_{u}, u \in \mathcal{I}_{t}$ and the $[W]_{u}, u \in \mathcal{I}_{t}$ are independent of $\mathcal{H}_{t}$, see Theorem 4.14 in [18]. Since we do not assume $\mathbb{E}\left[\left|W_{t}\right|^{3}\right]<\infty$, we start by investigating the sums as above with truncated summands. For $t \geq 0$ and $r \in(0, \infty]$, let

$$
W_{t, r}(u):=e^{-\alpha S(u)}\left(\left[W_{r+t-.}\right]_{u} \circ S(u)-1\right) \mathbb{1}_{\left\{e^{\alpha t / 2} e^{-\alpha S(u)}\left|\left[W_{r+t-.}\right]_{u} \circ S(u)-1\right| \leq 1\right\}}
$$

and

$$
V_{t, r}=\sum_{u \in \mathcal{I}_{t}}\left(W_{t, r}(u)-\mathbb{E}_{t}\left[W_{t, r}(u)\right]\right)
$$




\section{Gaussian fluctuations of Nerman's martingale}

Lemma 3.7. For $r \in(0, \infty]$, with $c_{r}$ as defined in (3.12), we have

$$
\lim _{t \rightarrow \infty} e^{\alpha t} \operatorname{Var}_{t}\left[V_{t, r}\right]=c_{r} W \text { a.s. }
$$

Proof. Conditionally on $\mathcal{H}_{t}$, the random variables $W_{t, r}(u), u \in \mathcal{I}_{t}$ are independent (but not identically distributed). By definition of $V_{t, r}$, we have

$$
\operatorname{Var}_{t}\left[V_{t, r}\right]=\sum_{u \in \mathcal{I}_{t}} \mathbb{E}_{t}\left[W_{t, r}(u)^{2}\right]-\sum_{u \in \mathcal{I}_{t}}\left(\mathbb{E}_{t}\left[W_{t, r}(u)\right]\right)^{2}=: G_{t, r}^{\prime}-G_{t, r}^{\prime \prime}
$$

It is sufficient to show that

$$
\begin{aligned}
& \lim _{t \rightarrow \infty} e^{\alpha t} G_{t, r}^{\prime}=c_{r} W \quad \text { a.s. } \\
& \text { and } \lim _{t \rightarrow \infty} e^{\alpha t} G_{t, r}^{\prime \prime}=0 \text { a.s. }
\end{aligned}
$$

To this end, for $t \in \mathbb{R}$, let $F_{t}$ and $G_{t}$ denote the distribution functions of $\left|W_{t}-1\right|$ and $\sup _{0 \leq s \leq t}\left|W_{s}-1\right|$, respectively. For instance, $F_{t}(x)=\mathbb{P}\left(\left|W_{t}-1\right| \leq x\right)$ for $x \in \mathbb{R}$.

Proof of (3.29). We have

$$
G_{t, r}^{\prime}=\sum_{u \in \mathcal{I}_{t}} \mathbb{E}_{t}\left[W_{t, r}(u)^{2}\right]=\sum_{u \in \mathcal{I}_{t}} e^{-2 \alpha S(u)} \int_{\left[0, e^{-\alpha t / 2} e^{\alpha S(u)}\right]} x^{2} \mathrm{~d} F_{r+t-S(u)}(x) .
$$

For $u \in \mathcal{I}_{t}, S(u)>t$ and therefore, for every $c>0, e^{-\alpha t / 2} e^{\alpha S(u)} \geq e^{\alpha t / 2} \geq c$ for all sufficiently large $t$. Consequently

$$
\sum_{u \in \mathcal{I}_{t}} e^{-2 \alpha S(u)} v_{r+t-S(u)}(c) \leq G_{t, r}^{\prime} \leq \sum_{u \in \mathcal{I}_{t}} e^{-2 \alpha S(u)} v_{r+t-S(u)}
$$

where $v_{s}(c):=\int_{[0, c]} x^{2} \mathrm{~d} F_{s}(x)$. Corollary 3.2 yields

$$
\begin{aligned}
e^{\alpha t} G_{t, r}^{\prime} & \leq e^{\alpha t} \sum_{u \in \mathcal{I}_{t}} e^{-2 \alpha S(u)} v_{r+t-S(u)} \\
& \rightarrow \frac{W}{-m^{\prime}(\alpha)} \mathbb{E}\left[\sum_{k=1}^{N} e^{-2 \alpha X_{k}} \int_{0}^{X_{k}} e^{\alpha x} v_{r+x-X_{k}} \mathrm{~d} x\right]=c_{r} W
\end{aligned}
$$

a.s. and in $L^{1}$ as $t \rightarrow \infty$ where the definition of $c_{r}$ should be recalled from (3.12). Analogously, for any $c>0$,

$$
\begin{aligned}
e^{\alpha t} G_{t, r}^{\prime} & \geq e^{\alpha t} \sum_{u \in \mathcal{I}_{t}} e^{-2 \alpha S(u)} v_{r+t-S(u)}(c) \\
& \rightarrow \frac{W}{-m^{\prime}(\alpha)} \mathbb{E}\left[\sum_{k=1}^{N} e^{-2 \alpha X_{k}} \int_{0}^{X_{k}} e^{\alpha x} v_{r+x-X_{k}}(c) \mathrm{d} x\right]
\end{aligned}
$$

a. s. and in $L^{1}$. Since $v_{s}(c) \uparrow v_{s}$ as $c \uparrow \infty$, (3.29) follows from the monotone convergence theorem.

Proof of (3.30). Since $\mathbb{E}\left[\left[W_{s}\right]_{u}-1\right]=0$ for all $s \in \mathbb{R}$,

$$
\begin{aligned}
G_{t, r}^{\prime \prime} & =\sum_{u \in \mathcal{I}_{t}} e^{-2 \alpha S(u)}\left(\mathbb{E}\left(\left(\left[W_{r+t-.}\right]_{u} \circ S(u)-1\right) \mathbb{1}_{\left\{e^{\alpha t / 2} e^{-\alpha S(u)}\left|\left[W_{r+t-} .\right]_{u} \circ S(u)-1\right| \leq 1\right\}}\right)\right)^{2} \\
& =\sum_{u \in \mathcal{I}_{t}} e^{-2 \alpha S(u)}\left(\mathbb{E}\left[\left(\left[W_{r+t-.}\right]_{u} \circ S(u)-1\right) \mathbb{1}_{\left.\left\{e^{\alpha t / 2} e^{-\alpha S(u)} \mid\left[W_{r+t-.}\right]\right]_{u} \circ S(u)-1 \mid>1\right\}}\right]\right)^{2} .
\end{aligned}
$$




\section{Gaussian fluctuations of Nerman's martingale}

In view of $\left[W_{s}\right]_{u}-1 \leq\left|\left[W_{s}\right]_{u}-1\right|$ and $S(u)>t$ for $u \in \mathcal{I}_{t}$, we have

$$
\begin{aligned}
G_{t, r}^{\prime \prime} & \leq \sum_{u \in \mathcal{I}_{t}}\left(e^{-2 \alpha S(u)}\left(\int_{\left(e^{-\alpha t / 2} e^{\alpha S(u)}, \infty\right)} x \mathrm{~d} F_{r+t-S(u)}(x)\right)^{2}\right) \\
& \leq \sum_{u \in \mathcal{I}_{t}} e^{-2 \alpha S(u)}\left(\int_{(c, \infty)} x \mathrm{~d} F_{r+t-S(u)}(x)\right)^{2} \\
& =: \sum_{u \in \mathcal{I}_{t}} e^{-2 \alpha S(u)} \bar{v}_{r+t-S(u)}(c)
\end{aligned}
$$

for all sufficiently large $t$ where $\bar{v}_{x}(c):=\left(\mathbb{E}\left[\left|W_{x}-1\right| \mathbb{1}_{\left\{\left|W_{x}-1\right| \geq c\right\}}\right]\right)^{2}$ for $x \geq 0$. Since $\left(\left(W_{t}-1\right)^{2}\right)_{t \geq 0}$ is a submartingale,

$$
0 \leq \bar{v}_{t}(c) \leq \mathbb{E}\left[\left(W_{t}-1\right)^{2}\right] \leq \mathbb{E}\left[(W-1)^{2}\right]=\sigma_{W}^{2}<\infty,
$$

i.e., $t \mapsto \bar{v}_{t}(c)$ is a nonnegative and bounded function. Doob's maximal inequality enables us to apply the dominated convergence theorem to show that the function $t \mapsto \bar{v}_{t}(c)$ is càdlàg. Consequently, we may apply (3.7) to conclude that

$$
\begin{aligned}
e^{\alpha t} G_{t, r}^{\prime \prime} & \leq \sum_{u \in \mathcal{I}_{t}} e^{-2 \alpha S(u)} \bar{v}_{r+t-S(u)}(c) \\
& \rightarrow \frac{W}{-m^{\prime}(\alpha)} \mathbb{E}\left[\sum_{k=1}^{N} e^{-2 \alpha X_{k}} \int_{0}^{X_{k}} e^{\alpha x} \bar{v}_{r+x-X_{k}}(c) \mathrm{d} x\right] \quad \text { a.s. and in } L^{1} .
\end{aligned}
$$

Further,

$$
\|\bar{v} \cdot(c)\|_{\infty}:=\sup _{t \in \mathbb{R}} v_{t}(c)=\sup _{t \in \mathbb{R}} \mathbb{E}\left[\left|W_{t}-1\right| \mathbb{1}_{\left\{\left|W_{t}-1\right| \geq c\right\}}\right] \rightarrow 0 \quad \text { as } c \rightarrow \infty .
$$

Therefore, as $c \rightarrow \infty$,

$$
\begin{aligned}
\mathbb{E}\left[\sum_{k=1}^{N} e^{-2 \alpha X_{k}} \int_{0}^{X_{k}} e^{\alpha x} \bar{v}_{r+x-X_{k}}(c) \mathrm{d} x\right] & =\int_{[0, \infty)} e^{-2 \alpha t} \int_{0}^{t} e^{\alpha x} \bar{v}_{r+x-t}(c) \mathrm{d} x \mu(\mathrm{d} t) \\
& \leq \frac{\|\bar{v} \cdot(c)\|_{\infty}}{\alpha} \int_{[0, \infty)} e^{-2 \alpha t}\left(e^{\alpha t}-1\right) \mu(\mathrm{d} t) \rightarrow 0
\end{aligned}
$$

This proves (3.30).

Our proof of (2.14) consists of two parts. In the first part, Lemma 3.8, we obtain (2.14) with the limit $t \rightarrow \infty$ taken along the points of a lattice $\delta n, n \in \mathbb{N}$ where $\delta>0$ is fixed but arbitrary. In the second part, we extend the convergence in (2.14) along lattice sequences to arbitrary sequences $t \rightarrow \infty$.

Lemma 3.8. For every $\delta>0$, we have

$$
\limsup _{n \rightarrow \infty} \frac{e^{\alpha n \delta / 2}}{\sqrt{\log (n \delta)}}\left(W-W_{n \delta}\right)=\left(\frac{2 \sigma^{2}}{-\alpha m^{\prime}(\alpha)} W\right)^{1 / 2} \text { a.s. }
$$

Proof. Fix an arbitrary $\delta>0$ and $r \in \delta \mathbb{N} \cup\{\infty\}$. We claim that (3.25) holds for the random variables

$$
T_{n}:=V_{\delta n, r} / \sqrt{\operatorname{Var}_{\delta n}\left[V_{\delta n, r}\right]}, \quad n \in \mathbb{N}_{0}
$$




\section{Gaussian fluctuations of Nerman's martingale}

and $\mathcal{R}_{n}=\mathcal{H}_{\delta n}$. Conditionally given $\mathcal{H}_{\delta n}, V_{\delta n, r}$ is a weighted sum of independent, centered random variables to which Lemma 3.6 applies. In particular, (3.26) yields

$$
\begin{aligned}
\sup _{y \in \mathbb{R}} \mid & \mathbb{P}\left(\frac{V_{\delta n, r}}{\sqrt{\operatorname{Var}_{\delta n}\left[V_{\delta n, r}\right]}} \leq y \mid \mathcal{H}_{\delta n}\right)-\Phi(y) \mid \\
\leq & C \frac{\sum_{u \in \mathcal{I}_{\delta n}} \mathbb{E}_{\delta n}\left[\left|W_{\delta n, r}(u)-\mathbb{E}_{\delta n}\left[W_{\delta n, r}(u)\right]\right|^{3}\right]}{\left(\operatorname{Var}_{\delta n}\left[V_{\delta n, r}\right]\right)^{3 / 2}} \\
& \leq 8 C \frac{\sum_{u \in \mathcal{I}_{\delta n}} \mathbb{E}_{\delta n}\left[\left|W_{\delta n, r}(u)\right|^{3}\right]}{\left(\operatorname{Var}_{\delta n}\left[V_{\delta n, r}\right]\right)^{3 / 2}}
\end{aligned}
$$

where $C>0$ is a finite absolute constant. In view of (3.28), the condition (3.25) will follow from the almost sure finiteness of

$$
I:=\sum_{n \geq 0} e^{3 \alpha \delta n / 2} \sum_{u \in \mathcal{I}_{\delta n}} \mathbb{E}_{\delta n}\left[\left|W_{\delta n, r}(u)\right|^{3}\right] .
$$

Here, $\mathbb{E}_{\delta n}\left[\left|W_{\delta n, r}(u)\right|^{3}\right]=e^{-3 \alpha S(u)} \int_{[0, \infty)} x^{3} \mathbb{1}_{\left\{e^{-\alpha \delta n / 2} e^{\alpha S(u)} \geq x\right\}} \mathrm{d} F_{r+\delta n-S(u)}(x)$. If $S(u)>$ $\delta n+r$, then $F_{r+\delta n-S(u)}$ is the distribution function of the Dirac measure at 0 and hence the integral vanishes. Otherwise, since $F_{t} \geq G_{t} \geq G_{\infty}$ pointwise for any $t>0$, we may estimate

$$
\begin{gathered}
\int_{[0, \infty)} e^{3 \alpha \delta n / 2-3 \alpha S(u)} x^{3} \mathbb{1}_{\left\{e^{-\alpha \delta n / 2} e^{\alpha S(u)} \geq x\right\}} \mathrm{d} F_{r+\delta n-S(u)}(x) \\
\leq \int_{[0, \infty)} \min \left\{e^{3 \alpha \delta n / 2-3 \alpha S(u)} x^{3}, 1\right\} \mathrm{d} F_{r+\delta n-S(u)}(x) \\
\leq \int_{[0, \infty)} \min \left\{e^{3 \alpha \delta n / 2-3 \alpha S(u)} x^{3}, 1\right\} \mathrm{d} G_{\infty}(x) .
\end{gathered}
$$

As in Section 2.3, denote by $\left(\mathrm{S}_{n}\right)_{n \in \mathbb{N}_{0}}$ a random walk with i.i.d. increments $\mathrm{S}_{n}-\mathrm{S}_{n-1}$, $n \in \mathbb{N}$ and increment law $\mathbb{P}\left(\mathrm{S}_{n}-\mathrm{S}_{n-1} \in \mathrm{d} x\right):=e^{-\alpha x} \mu(\mathrm{d} x)$. By the many-to-one lemma [25, Section 1.3],

$$
\begin{aligned}
\mathbb{E}[I] & \leq \int_{[0, \infty)} \mathbb{E}\left[\sum_{n \geq 0} \sum_{u \in \mathcal{I}_{\delta n}} \min \left\{e^{3 \alpha \delta n / 2-3 \alpha S(u)} x^{3}, 1\right\}\right] \mathrm{d} G_{\infty}(x) \\
& =\int_{[0, \infty)} \mathbb{E}\left[\sum_{n \geq 0} \min \left\{e^{3 \alpha \delta n / 2-3 \alpha \mathrm{S}_{\tau \delta n}} x^{3}, 1\right\} e^{\alpha \mathrm{S}_{\tau_{\delta n}}}\right] \mathrm{d} G_{\infty}(x),
\end{aligned}
$$

where $\tau_{\delta n}=\inf \left\{k \in \mathbb{N}_{0}: \mathrm{S}_{k}>\delta n\right\}$ is the first-passage time of the level $\delta n$ of the random walk $\left(\mathrm{S}_{n}\right)_{n \in \mathbb{N}_{0}}$ for $n \in \mathbb{N}_{0}$. The integrand above can be estimated as follows

$$
\begin{gathered}
\sum_{n \geq 0} \min \left\{e^{3 \alpha \delta n / 2-3 \alpha \mathrm{S}_{\tau_{\delta n}}} x^{3}, 1\right\} e^{\alpha \mathrm{S}_{\tau_{\delta n}}} \leq \sum_{n \geq 0} \min \left\{e^{-3 \alpha \mathrm{S}_{\tau_{\delta n}} / 2} x^{3}, 1\right\} e^{\alpha \mathrm{S}_{\tau_{\delta n}}} \\
=x^{3} \sum_{n: \mathrm{S}_{\tau_{\delta n}} \geq \frac{2}{\alpha} \log x} e^{-\alpha \mathrm{S}_{\tau_{\delta n}} / 2}+\sum_{n: \mathrm{S}_{\tau_{\delta n}} \leq \frac{2}{\alpha} \log x} e^{\alpha \mathrm{S}_{\tau_{\delta n}}} .
\end{gathered}
$$

Let $\mathrm{X}$ be a copy of $S_{1}$ independent of $\left(S_{n}\right)_{n \in \mathbb{N}_{0}}$. Since for any $i \in \mathbb{N}$ the set $\left\{n \in \mathbb{N}_{0}\right.$ : $\left.\tau_{\delta n}=i\right\}$ has cardinality at most $\delta^{-1}\left(\mathrm{~S}_{i}-\mathrm{S}_{i-1}\right)+1$, for any real $y$ and $\beta>0$, we may estimate

$$
\begin{aligned}
\mathbb{E}\left[\sum_{n: \mathrm{S}_{\tau_{\delta n}} \geq y} e^{-\beta \mathrm{S}_{\tau_{\delta n}}}\right] & \leq \mathbb{E}\left[\sum_{i \geq 1} \mathbb{1}_{\left\{\mathrm{S}_{i} \geq y\right\}} e^{-\beta \mathrm{S}_{i}}\left(\delta^{-1}\left(\mathrm{~S}_{i}-\mathrm{S}_{i-1}\right)+1\right)\right] \\
& =e^{-\beta y} \mathbb{E}\left[\sum_{i \geq 0} \mathbb{1}_{\left\{\mathrm{S}_{i}+\mathrm{X}-y \geq 0\right\}} e^{-\beta\left(\mathrm{S}_{i}+\mathrm{X}-y\right)}\left(\delta^{-1} \mathbf{X}+1\right)\right] .
\end{aligned}
$$




\section{Gaussian fluctuations of Nerman's martingale}

Now let $f(x):=e^{\beta x} \mathbb{1}_{(-\infty, 0]}(x)$ for $x \in \mathbb{R}$, and let $\mathrm{U}$ denote the renewal measure associated with $\left(\mathrm{S}_{n}\right)_{n \in \mathbb{N}_{0}}$, i.e., $\mathrm{U}(B)=\sum_{n \in \mathbb{N}_{0}} \mathbb{P}\left(S_{n} \in B\right)$ for Borel sets $B \subseteq \mathbb{R}$. The function $f$ is directly Riemann integrable, hence $f * \mathrm{U}(x):=\int f(x-u) \mathrm{U}(\mathrm{d} u)$ is bounded by some finite constant $C>0$ (that may depend on $\beta$ ) by the key renewal theorem. Consequently,

$$
\begin{array}{rl}
e^{-\beta y} & \mathbb{E}\left[\sum_{i \geq 0} \mathbb{1}_{\left\{\mathrm{S}_{i}+\mathrm{X}-y \geq 0\right\}} e^{-\beta\left(\mathrm{S}_{i}+\mathbf{X}-y\right)}\left(\delta^{-1} \mathbf{X}+1\right)\right] \\
& =e^{-\beta y} \mathbb{E}\left[f * \mathrm{U}(y-x)\left(\delta^{-1} \mathbf{X}+1\right)\right] \\
& \leq C e^{-\beta y} \mathbb{E}\left[\delta^{-1} \mathbf{X}+1\right]=C\left(-\delta^{-1} m^{\prime}(\alpha)+1\right) e^{-\beta y}
\end{array}
$$

Similarly, by increasing the value of $C>0$ if necessary,

$$
\begin{aligned}
\mathbb{E}\left[\sum_{n: \mathrm{S}_{\tau_{\delta n}} \leq y} e^{\beta \mathrm{S}_{\tau_{\delta n}}}\right] & \leq \mathbb{E}\left[\sum_{i \geq 1} \mathbb{1}_{\left\{\mathrm{S}_{i} \leq y\right\}} e^{\beta \mathrm{S}_{i}}\left(\delta^{-1}\left(\mathrm{~S}_{i}-\mathrm{S}_{i-1}\right)+1\right)\right] \\
& =e^{\beta y} \mathbb{E}\left[\sum_{i \geq 0} \mathbb{1}_{\left\{\mathrm{S}_{i}+\mathrm{X}-y \leq 0\right\}} e^{\beta\left(\mathrm{S}_{i}+\mathrm{X}-y\right)}\left(\delta^{-1} \mathbf{X}+1\right)\right] \\
& \leq C e^{\beta y} \mathbb{E}\left[\delta^{-1} \mathbf{X}+1\right]=C\left(-\delta^{-1} m^{\prime}(\alpha)+1\right) e^{\beta y}
\end{aligned}
$$

Hence, for some constant $C^{\prime}>0$,

$$
\mathbb{E}\left[\sum_{n \geq 0} \min \left\{e^{3 \alpha \delta n / 2-3 \alpha S_{\tau \delta n}} x^{3}, 1\right\} e^{\alpha \mathrm{S}_{\tau \delta n}}\right] \leq C x^{2},
$$

which, in turn gives,

$$
\mathbb{E}[I] \leq C \int_{[0, \infty)} x^{2} \mathrm{~d} G_{\infty}(x)=C \mathbb{E}\left[\sup _{s \geq 0}\left(W_{s}-1\right)^{2}\right]<\infty
$$

in view of (3.5). In particular, $I<\infty$ almost surely and the condition (3.25) is fulfilled. An appeal to Lemma 3.5 with $T_{n}=V_{\delta n, r} / \sqrt{\operatorname{Var}_{\delta n}\left[V_{\delta n, r}\right]}$ in combination with (3.28) gives, for fixed $r \in \delta \mathbb{N}$,

$$
\limsup _{n \rightarrow \infty} \sqrt{\frac{e^{\alpha \delta n}}{\log (\delta n)}} V_{\delta n, r}=\sqrt{2 c_{r} W} \text { a.s. }
$$

because $V_{\delta n, r}$ is $\mathcal{H}_{\delta n+r}$-measurable; whereas

$$
\limsup _{n \rightarrow \infty} \sqrt{\frac{e^{\alpha \delta n}}{\log (\delta n)}} V_{\delta n, \infty} \leq \sqrt{2 c_{\infty} W}=\left(\frac{2 \sigma^{2}}{-\alpha m^{\prime}(\alpha)} W\right)^{1 / 2} \text { a.s. }
$$

Next, we shall prove that (3.35) and (3.36) entail

$$
\limsup _{n \rightarrow \infty} \sqrt{\frac{e^{\alpha \delta n}}{\log (\delta n)}}\left(W_{\delta n+r}-W_{\delta n}\right)=\sqrt{2 c_{r} W} \text { a.s. }
$$

for fixed $r \in \delta \mathbb{N}$ and

$$
\limsup _{n \rightarrow \infty} \sqrt{\frac{e^{\alpha \delta n}}{\log (\delta n)}}\left(W-W_{\delta n}\right) \leq \sqrt{2 c_{\infty} W}=\left(\frac{2 \sigma^{2}}{-\alpha m^{\prime}(\alpha)} W\right)^{1 / 2} \text { a.s. }
$$

To this end, it is enough to check that, for $r \in \delta \mathbb{N} \cup\{\infty\}$,

$$
\begin{aligned}
& \lim _{n \rightarrow \infty} e^{\alpha \delta n / 2} \sum_{u \in \mathcal{I}_{\delta n}} e^{-\alpha S(u)}\left|\left[W_{r+\delta n-.}\right]_{u} \circ S(u)-1\right| \\
& \cdot \mathbb{1}_{\left\{e^{\alpha \delta n / 2} e^{-\alpha S(u)}\left|\left[W_{r+\delta n-.}\right]_{u} \circ S(u)-1\right|>1\right\}}=0 \text { a.s. }
\end{aligned}
$$




\section{Gaussian fluctuations of Nerman's martingale}

and

$$
\lim _{n \rightarrow \infty} e^{\alpha \delta n / 2} \sum_{u \in \mathcal{I}_{\delta n}}\left|\mathbb{E}_{\delta n}\left[W_{\delta n, r}(u)\right]\right|=0 \quad \text { a.s. }
$$

Since, for $u \in \mathcal{I}_{\delta n}, \mathbb{E}_{\delta n}\left[\left[W_{r}\right]_{u}-1\right]=0$ and $S(u)$ is $\mathcal{H}_{\delta n}$-measurable, we have

$$
\begin{aligned}
\mid \mathbb{E}_{\delta n} & {\left[W_{\delta n, r}(u)\right] \mid } \\
& =\left|\mathbb{E}_{\delta n}\left[e^{-\alpha S(u)}\left(\left[W_{r+\delta n-.}\right]_{u} \circ S(u)-1\right) \mathbb{1}_{\left\{e^{\alpha \delta n / 2} e^{-\alpha S(u)}\left|\left[W_{r+\delta n-.}\right]_{u} \circ S(u)-1\right| \leq 1\right\}}\right]\right| \\
& =\left|\mathbb{E}_{\delta n}\left[e^{-\alpha S(u)}\left(\left[W_{r+\delta n-.}\right]_{u} \circ S(u)-1\right) \mathbb{1}_{\left\{e^{\alpha \delta n / 2} e^{-\alpha S(u)}\left|\left[W_{r+\delta n-.}\right]_{u} \circ S(u)-1\right|>1\right\}}\right]\right| \\
& \leq \mathbb{E}_{\delta n}\left[e^{-\alpha S(u)}\left|\left[W_{r+\delta n-.}\right]_{u} \circ S(u)-1\right| \mathbb{1}_{\left\{e^{\alpha \delta n / 2} e^{-\alpha S(u)}\left|\left[W_{r+\delta n-.}\right]_{u} \circ S(u)-1\right|>1\right\}}\right] .
\end{aligned}
$$

Hence, both relations (3.39) and (3.40) follow if we can show that

$$
\mathbb{E}\left[\sum_{n \geq 0} e^{\alpha \delta n / 2} \sum_{u \in \mathcal{I}_{\delta n}} e^{-\alpha S(u)} \int_{\left(e^{-\alpha \delta n / 2} e^{\alpha S(u)}, \infty\right)} x \mathrm{~d} F_{r+\delta n-S(u)}(x)\right]<\infty
$$

To see this, notice that

$$
\begin{aligned}
\mathbb{E}\left[\sum_{n \geq 0} e^{\alpha \delta n / 2} \sum_{u \in \mathcal{I}_{\delta n}} e^{-\alpha S(u)} \int_{\left(e^{-\alpha \delta n / 2} e^{\alpha S(u)}, \infty\right)} x \mathrm{~d} F_{r+\delta n-S(u)}(x)\right] \\
\leq \mathbb{E}\left[\sum_{n \geq 0} e^{\alpha \delta n / 2} \sum_{u \in \mathcal{I}_{\delta n}} e^{-\alpha S(u)} \int_{\left(e^{\alpha(\delta n / 2+r)}, \infty\right)} x \mathrm{~d} G_{r}(x)\right] \\
=\sum_{n \geq 0} e^{\alpha \delta n / 2} \int_{\left(e^{\alpha(\delta n / 2+r)}, \infty\right)} x \mathrm{~d} G_{r}(x) \\
\leq \int_{(1, \infty)} x\left(\sum_{n=0}^{(2 / \delta)\left(\frac{\log x}{\alpha}-r\right)} e^{\alpha \delta n / 2}\right) \mathrm{d} G_{r}(x) \\
\leq \text { const } \cdot \int_{(1, \infty)} x^{2} \mathrm{~d} G_{r}(x)<\infty
\end{aligned}
$$

The proof of (3.37) and (3.38) is complete.

It remains to show that " $\leq$ " can be replaced by "=" in (3.38). As has already been remarked at the beginning of the proof, once we have proved (3.38), we also have

$$
\liminf _{n \rightarrow \infty} \sqrt{\frac{e^{\alpha \delta n}}{\log (\delta n)}}\left(W-W_{\delta n}\right) \geq-\left(\frac{2 \sigma^{2}}{-\alpha m^{\prime}(\alpha)} W\right)^{1 / 2} \text { a.s. }
$$

For any $r \in \delta \mathbb{N}$, the following equality holds

$$
\begin{gathered}
\sqrt{\frac{e^{\alpha \delta n}}{\log (\delta n)}}\left(W-W_{\delta n}\right)=\sqrt{\frac{e^{\alpha(\delta n+r)}}{\log (\delta n+r)}}\left(W-W_{\delta n+r}\right) \sqrt{\frac{\log (\delta n+r)}{\log (\delta n)}} e^{-\alpha \delta r / 2} \\
+\sqrt{\frac{e^{\alpha \delta n}}{\log (\delta n)}}\left(W_{\delta n+r}-W_{\delta n}\right) .
\end{gathered}
$$




\section{Gaussian fluctuations of Nerman's martingale}

From (3.37) and (3.42) we infer

$$
\begin{aligned}
\limsup _{n \rightarrow \infty} & \sqrt{\frac{e^{\alpha \delta n}}{\log (\delta n)}}\left(W-W_{\delta n}\right) \\
\geq & \liminf _{n \rightarrow \infty} \sqrt{\frac{e^{\alpha(\delta n+r)}}{\log (\delta n+r)}}\left(W-W_{\delta n+r}\right) \sqrt{\frac{\log (\delta n+r)}{\log (\delta n)}} e^{-\alpha \delta r / 2} \\
& +\limsup _{n \rightarrow \infty} \sqrt{\frac{e^{\alpha \delta n}}{\log (\delta n)}}\left(W_{\delta n+r}-W_{\delta n}\right) \\
\geq & -\left(\frac{2 \sigma^{2}}{-\alpha m^{\prime}(\alpha)} W\right)^{1 / 2} e^{-\alpha \delta r / 2}+\left(2 c_{r} W\right)^{1 / 2} .
\end{aligned}
$$

Letting $r \rightarrow \infty$, we arrive at

$$
\limsup _{n \rightarrow \infty} \sqrt{\frac{e^{\alpha \delta n}}{\log (\delta n)}}\left(W-W_{\delta n}\right) \geq\left(2 c_{\infty} W\right)^{1 / 2}=\left(\frac{2 \sigma^{2}}{-\alpha m^{\prime}(\alpha)}\right)^{1 / 2} \text { a.s. }
$$

Proof of Theorem 2.3. We have to show that (2.14) with $\delta n$ replacing $t$ entails (2.14). Plainly,

$$
\limsup _{t \rightarrow \infty} \sqrt{\frac{e^{\alpha t}}{\log t}}\left(W-W_{t}\right) \geq \limsup _{n \rightarrow \infty} \sqrt{\frac{e^{\alpha \delta n}}{\log (\delta n)}}\left(W-W_{\delta n}\right)=\left(\frac{2 \sigma^{2}}{-\alpha m^{\prime}(\alpha)} W\right)^{1 / 2} \text { a.s. }
$$

Thus, it remains to prove the converse inequality

$$
\limsup _{t \rightarrow \infty} \sqrt{\frac{e^{\alpha t}}{\log t}}\left(W-W_{t}\right) \leq\left(\frac{2 \sigma^{2}}{-\alpha m^{\prime}(\alpha)} W\right)^{1 / 2} \text { a.s. }
$$

To this end, fix arbitrary $\delta, \rho>0$, let $n \in \mathbb{N}_{0}$ and notice that the following particular case of (3.37) holds

$$
\limsup _{n \rightarrow \infty} \sqrt{\frac{e^{\alpha \delta n}}{\log (\delta n)}}\left(W_{\delta n}-W_{\delta(n+1)}\right)=\sqrt{2 c_{\delta} W} \text { a.s. }
$$

This in combination with the fact that $W_{\delta n}-W_{\delta(n+1)}$ is $\mathcal{H}_{\delta(n+1)}$-measurable and the conditional Borel-Cantelli lemma (see, for instance, Theorem 5.3.2 on p. 240 in [7]) implies

$$
\sum_{n \geq 1} \mathbb{P}\left(W_{\delta n}-W_{\delta(n+1)}>\varepsilon_{n} \mid \mathcal{H}_{\delta n}\right)<\infty \text { a.s. }
$$

for

$$
\varepsilon_{n}=(1+\rho) \sqrt{\frac{\log (\delta n)}{e^{\alpha \delta n}}} \sqrt{2 c_{\delta} W_{\delta n}} .
$$

For $t \in[\delta n, \delta(n+1))$, define

$$
A_{t}:=\mathbb{E}_{t}\left[\left(W_{\delta(n+1)}-W_{t}\right)^{2}\right]
$$

and observe that, in view of Lemma 3.1 and the fact that the martingale $\left(W_{t}\right)_{t \geq 0}$ is $L^{2}$-bounded,

$$
\sup _{t \geq 0} e^{\alpha t} A_{t}=\sup _{t \geq 0} e^{\alpha t} \sum_{u \in \mathcal{I}_{t}} e^{-2 \alpha S(u)} v_{\delta(n+1)-S(u)}<\infty \quad \text { a.s. }
$$

Now let

$$
B_{n}:=\sup _{t \in[\delta n, \delta(n+1))}\left(W_{\delta n}-W_{t}-\left(2 A_{t}\right)^{1 / 2}\right)
$$




\section{Gaussian fluctuations of Nerman's martingale}

and

$$
t_{n}^{*}:=\inf \left\{s \geq \delta n: W_{\delta n}-W_{s}-\left(2 A_{s}\right)^{1 / 2}>\varepsilon_{n}\right\} .
$$

Then $t_{n}^{*}$, being the hitting time of an open set by an adapted, right-continuous process, is an optional time for $\left(\mathcal{H}_{t}\right)_{t \geq 0}$. Thus,

$$
\begin{aligned}
& \mathbb{P}\left(W_{\delta n}-W_{\delta(n+1)}>\varepsilon_{n} \mid \mathcal{H}_{\delta n}\right) \geq \mathbb{P}\left(W_{\delta n}-W_{\delta(n+1)}>\varepsilon_{n}, t_{n}^{*}<\delta(n+1) \mid \mathcal{H}_{\delta n}\right) \\
& \quad \geq \mathbb{P}\left(W_{t_{n}^{*}}-W_{\delta(n+1)}>-\left(2 A_{t_{n}^{*}}\right)^{1 / 2}, t_{n}^{*}<\delta(n+1) \mid \mathcal{H}_{\delta n}\right) \\
& \quad=\mathbb{E}\left[\mathbb{P}\left(W_{t_{n}^{*}}-W_{\delta(n+1)}>-\left(2 A_{t_{n}^{*}}\right)^{1 / 2} \mid \mathcal{H}_{t_{n}^{*}}\right) \mathbb{1}_{\left\{t_{n}^{*}<\delta(n+1)\right\}} \mid \mathcal{H}_{\delta n}\right] \\
& \quad \geq 2^{-1} \mathbb{P}\left(t_{n}^{*}<\delta(n+1) \mid \mathcal{H}_{\delta n}\right)=2^{-1} \mathbb{P}\left(B_{n}>\varepsilon_{n} \mid \mathcal{H}_{\delta n}\right)
\end{aligned}
$$

having used the definition of $t_{n}^{*}$ and the fact that $t_{n}^{*}$ is optional for the second inequality, the tower property of the conditional expectations for the first equality and the Markov inequality for the last inequality.

This entails

$$
\sum_{n \geq 1} \mathbb{P}\left(B_{n}>\varepsilon_{n} \mid \mathcal{H}_{\delta n}\right)<\infty \text { a.s. }
$$

and thereupon $B_{n} \leq \varepsilon_{n}$ eventually a.s. Thus,

$$
\begin{aligned}
& \limsup _{t \rightarrow \infty} \sqrt{\frac{e^{\alpha t}}{\log t}}\left(W-W_{t}\right) \\
& \leq e^{\alpha \delta / 2}\left(\limsup _{n \rightarrow \infty} \sqrt{\frac{e^{\alpha \delta n}}{\log (\delta n)}}\left(W-W_{\delta n}\right)+\limsup _{n \rightarrow \infty} \sqrt{\frac{e^{\alpha \delta n}}{\log (\delta n)}} \sup _{t \in[\delta n, \delta(n+1))}\left(W_{\delta n}-W_{t}\right)\right) \\
& =e^{\alpha \delta / 2}\left(\left(\frac{2 \sigma^{2}}{-\alpha m^{\prime}(\alpha)} W\right)^{1 / 2}+\limsup _{n \rightarrow \infty} \sqrt{\frac{e^{\alpha \delta n}}{\log (\delta n)}} B_{n}\right) \\
& \leq e^{\alpha \delta / 2}\left(\left(\frac{2 \sigma^{2}}{-\alpha m^{\prime}(\alpha)} W\right)^{1 / 2}+(1+\rho) \sqrt{2 c_{\delta} W}\right),
\end{aligned}
$$

where the equality is a consequence of (3.46). Recall the definition of $c_{\delta}$ from (3.31) and notice that $\lim _{\delta \rightarrow 0+} c_{\delta}=0$ by Corollary 3.2. With this at hand, letting $\delta \downarrow 0$ gives (3.43).

\section{References}

[1] Gerold Alsmeyer and Dirk Kuhlbusch, Double martingale structure and existence of $\phi$ moments for weighted branching processes, Münster J. Math. 3 (2010), 163-211. MR2775360

[2] Søren Asmussen and Heinrich Hering, Branching processes, Progress in Probability and Statistics, vol. 3, Birkhäuser Boston, Inc., Boston, MA, 1983. MR701538

[3] John D. Biggins, Uniform convergence of martingales in the branching random walk, Ann. Probab. 20 (1992), no. 1, 137-151. MR1143415

[4] John D. Biggins and Andreas E. Kyprianou, Measure change in multitype branching, Adv. in Appl. Probab. 36 (2004), no. 2, 544-581. MR2058149

[5] Patrick Billingsley, Convergence of probability measures, second ed., Wiley Series in Probability and Statistics: Probability and Statistics, John Wiley \& Sons, Inc., New York, 1999, A Wiley-Interscience Publication. MR1700749

[6] Dariusz Buraczewski, Alexander Iksanov, and Bastien Mallein, On the derivative martingale in a branching random walk, Ann. Probab. 49 (2021), no. 3, 1164-1204. MR4255141

[7] Rick Durrett, Probability: theory and examples, fourth ed., Cambridge Series in Statistical and Probabilistic Mathematics, vol. 31, Cambridge University Press, Cambridge, 2010. MR2722836 
[8] Dimitris Gatzouras, On the lattice case of an almost-sure renewal theorem for branching random walks, Adv. in Appl. Probab. 32 (2000), no. 3, 720-737. MR1788092

[9] Inge S. Helland, Central limit theorems for martingales with discrete or continuous time, Scand. J. Statist. 9 (1982), no. 2, 79-94. MR668684

[10] Christopher C. Heyde, A rate of convergence result for the super-critical Galton-Watson process, J. Appl. Probability 7 (1970), 451-454. MR0288859

[11] Christopher C. Heyde, Some central limit analogues for supercritical Galton-Watson processes, J. Appl. Probability 8 (1971), 52-59. MR282422

[12] Christopher C. Heyde and Bruce M. Brown, An invariance principle and some convergence rate results for branching processes, Z. Wahrscheinlichkeitstheorie und Verw. Gebiete 20 (1971), 271-278. MR0310987

[13] Alexander Iksanov and Zakhar Kabluchko, A central limit theorem and a law of the iterated logarithm for the Biggins martingale of the supercritical branching random walk, J. Appl. Probab. 53 (2016), no. 4, 1178-1192. MR3581250

[14] Alexander Iksanov, Konrad Kolesko, and Matthias Meiners, Stable-like fluctuations of Biggins' martingales, Stochastic Process. Appl. 129 (2019), no. 11, 4480-4499. MR4013869

[15] Alexander Iksanov, Konrad Kolesko, and Matthias Meiners, Fluctuations of Biggins' martingales at complex parameters, Ann. Inst. Henri Poincaré Probab. Stat. 56 (2020), no. 4, 2445-2479. MR4164844

[16] Alexander Iksanov and Matthias Meiners, Rate of convergence in the law of large numbers for supercritical general multi-type branching processes, Stochastic Process. Appl. 125 (2015), no. 2, 708-738. MR3293300

[17] Peter Jagers, Branching processes with biological applications, Wiley-Interscience [John Wiley \& Sons], London-New York-Sydney, 1975, Wiley Series in Probability and Mathematical Statistics-Applied Probability and Statistics. MR0488341

[18] Peter Jagers, General branching processes as Markov fields, Stochastic Process. Appl. 32 (1989), no. 2, 183-212. MR1014449

[19] Svante Janson, Asymptotics of fluctuations in Crump-Mode-Jagers processes: the lattice case, Adv. in Appl. Probab. 50 (2018), no. A, 141-171. MR3905097

[20] Quansheng Liu, On generalized multiplicative cascades, Stochastic Process. Appl. 86 (2000), no. 2, 263-286. MR1741808

[21] Pascal Maillard and Michel Pain, 1-stable fluctuations in branching Brownian motion at critical temperature I: The derivative martingale, Ann. Probab. 47 (2019), no. 5, 2953-3002. MR4021242

[22] Olle Nerman, On the convergence of supercritical general (C-M-J) branching processes, Z. Wahrsch. Verw. Gebiete 57 (1981), no. 3, 365-395. MR629532

[23] Yan-Xia Ren, Renming Song, Zhenyao Sun, and Jianjie Zhao, Stable central limit theorems for super Ornstein-Uhlenbeck processes, Electron. J. Probab. 24 (2019), Paper No. 141, 42 pp. MR4049077

[24] Uwe Rösler, Valentin A. Topchii, and Vladimir A. Vatutin, The rate of convergence for weighted branching processes [translation of Mat. Tr. 5 (2002), no. 1, 18-45; MR1918893 (2003g:60146)], Siberian Adv. Math. 12 (2002), no. 4, 57-82 (2003). MR1984638

[25] Zhan Shi, Branching random walks, Lecture Notes in Mathematics, vol. 2151, Springer, Cham, 2015, Lecture notes from the 42nd Probability Summer School held in Saint Flour, 2012, École d’Été de Probabilités de Saint-Flour. [Saint-Flour Probability Summer School]. MR3444654

Acknowledgments. The authors thank the Associate Editor and the anonymous referee for several useful comments. This work was initiated during the visit of A. Iksanov to Innsbruck in August 2019. He gratefully acknowledges hospitality and the financial support. 